\title{
APASL clinical practice guideline on hepatitis B reactivation related to the use of immunosuppressive therapy
}

\author{
George Lau ${ }^{1,2}$ - Ming-Lung $\mathrm{Yu}^{3} \cdot$ Grace Wong $^{4} \cdot$ Alexander Thompson $^{5} \cdot$ Hasmik Ghazinian $^{6} \cdot$ Jin-Lin Hou ${ }^{7}$. \\ Teerha Piratvisuth $^{8} \cdot \mathrm{Ji}^{-D o n g} \mathrm{Jia}^{9} \cdot$ Masashi Mizokami ${ }^{10}$. Gregory Cheng ${ }^{2,11}$. Guo-Feng Chen ${ }^{12}$. Zhen-Wen Liu ${ }^{13}$. \\ Oidov Baatarkhuu ${ }^{14}$. Ann Lii Cheng ${ }^{15}$. Woon Leung Ng ${ }^{16}$. Patrick Lau ${ }^{1}$. Tony Mok ${ }^{17}$. Jer-Ming Chang ${ }^{18}$. \\ Saeed Hamid ${ }^{19}$ - A. Kadir Dokmeci' ${ }^{20}$. Rino A. Gani ${ }^{21}$ - Diana A. Payawal ${ }^{22}$. Pierce Chow ${ }^{23}$. Joong-Won Park ${ }^{24}$. \\ Simone I. Strasser ${ }^{25} \cdot$ Rosmawaiti Mohamed $^{26} \cdot$ Khin Maung Win $^{27} \cdot$ Tanwandee Tawesak $^{28}$ - Shiv Kumar Sarin ${ }^{29}$. \\ Masao Omata ${ }^{30,31}$
}

Received: 29 March 2021 / Accepted: 20 July 2021 / Published online: 24 August 2021

(c) Asian Pacific Association for the Study of the Liver 2021

\begin{abstract}
Background \& Aim Hepatitis B reactivation related to the use of immunosuppressive therapy remains a major cause of liverrelated morbidity and mortality in hepatitis B endemic Asia-Pacific region. This clinical practice guidelines aim to assist clinicians in all disciplines involved in the use of immunosuppressive therapy to effectively prevent and manage hepatitis B reactivation.

Methods All publications related to hepatitis B reactivation with the use of immunosuppressive therapy since 1975 were reviewed. Advice from key opinion leaders in member countries/administrative regions of Asian-Pacific Association for the study of the liver was collected and synchronized. Immunosuppressive therapy was risk-stratified according to its reported rate of hepatitis $B$ reactivation.

Recommendations We recommend the necessity to screen all patients for hepatitis B prior to the initiation of immunosuppressive therapy and to administer pre-emptive nucleos(t)ide analogues to those patients with a substantial risk of hepatitis and acute-on-chronic liver failure due to hepatitis B reactivation.
\end{abstract}

Keywords APASL $\cdot$ Hepatitis B reactivation · Immunosuppressive therapy $\cdot$ Guideline

\section{Introduction}

More than four decades ago, based on serial measurement of hepatitis B virus (HBV) antigen and serum alanine aminotransferase (ALT) level, hepatitis due to HBV reactivation (HBVr) had been described in both hepatitis B surface antigen (HBsAg) positive and HBsAg negative but antibody (anti-HBs) positive patients with myeloproliferative and lymphoproliferative diseases treated with anti-tumor chemotherapy $[1,2]$. In the 90 s, prospective study showed that nearly half of the HBsAg-positive patients with malignant lymphoma treated with cytotoxic therapy, suffered

George Lau

gkklau@hnhmgl.com; gkklau@ netvigator.com

Ming-Lung Yu fish6069@gmail.com

Extended author information available on the last page of the article from hepatitis due to HBVr [3]. With the subsequent use of more aggressive immunosuppressive therapy (IST) such as the conditioning regimens in allogeneic hematopoietic stem cell therapy, fatality due to $\mathrm{HBVr}$ in $\mathrm{HBsAg}$ positive patients with hematological malignancy became an increasingly important clinical problem $[4,5]$. Hence, close monitoring of HBsAg-positive patients with malignancies receiving cytotoxic therapy was recommended [6]. Further progress in various medical disciplines with the use of more potent immunosuppressive therapy and targeted monoclonal therapy such as rituximab, a human/murine, chimeric antiCD20 monoclonal antibody, fatal fulminant hepatic failure due to $\mathrm{HBVr}$ was noted even in those who had recovered from past $\mathrm{HBV}$ infection-HBsAg negative but hepatitis B core antibody (anti-HBc) positive with HBV DNA detectable only by sensitive nested PCR [7-13]. At the turn of the twenty-first century, with the availability of potent nucleos(t) ide analogues (NUCs), pre-emptive use of lamivudine and 
then entecavir and tenofovir were shown in randomized controlled trials to be highly effective in preventing HBVr and its liver-related morbidity and mortality in both HBsAg positive and HBsAg negative but anti-HBs and anti-HBc positive patients treated with cytotoxic chemotherapy [13-16].

Up till 2020, numerous clinical guidelines had been formulated aiming to reduce the occurrence of hepatitis due to $\mathrm{HBVr}$ in patients treated with IST [17-21]. Nevertheless, hepatitis due to HBVr leading to acute on chronic liver failure remains a major health threat in Asia-Pacific region, where HBV infection is endemic [22]. The major barriers appear to be related to the non-compliance of medical practitioners in other non-hepatology disciplines. This is further compounded by the recent rapid expansion on the use of new immunosuppressive agents such as tyrosine-kinase inhibitors (TKIs), [23-26] immune checkpoint inhibitors (ICIs) [27-29] used in the treatment of various cancers and tumor necrosis factor (TNF) antagonists [30-35] for many autoimmune diseases. Recently, in those chronic hepatitis $\mathrm{C}$ (CHC) patients coinfected with hepatitis B, HBVr has also been reported during and after treatment with direct-acting antiviral agents (DAAs) [36-48].

The deterring factors for the successful implementation of the guidelines include lack of attention to the prevalence of $\mathrm{HBVr}$, unawareness of the ease of implementation of suitable preventive measures, miscalculations of the total costs to society and potentials for improvement in quality of care taking into consideration of the wide availability of potent generic NUCs at a very low cost and simple virological testing $[49,50]$. We aim to develop a user-friendly clinical practise guideline for all related medical disciplines which will help to curtail the morbidity and mortality related to $\mathrm{HBVr}$ in subjects treated with IST, especially in HBV endemic Asia-Pacific region.

\section{Methods}

With the initiation by the steering committee of AsianPacific Association for the study of the liver (APASL), a panel of experts from 21 different administrative regions/ countries in Asia-Pacific region was invited to form a working party which formulated this clinical practise guidance for HBVr in patients treated with IST. The panel includes not only hepatologists, but also oncologists, rheumatologists, transplant surgeons, nephrologists and interventional radiologists. The first working party meeting was conducted on 9th Nov 2019 in Boston during the American Association for the study of liver diseases (AASLD) annual meeting when the key questions related to $\mathrm{HBVr}$ were laid down, methods to develop the guidance were defined and drafting of recommendations was assigned. This guidance addresses the following questions: (1) What is the definition of HBVr?
(2) Who should be screened? (3) What should be done for screening? (4) How should a patient planned for IST be managed and monitored? All panel members were required to disclose their relationships with industry during the guidelines formulation until accepted for publication by Hepatology International (official journal of APASL). The Chair (G Lau) and Co-Chairs (ML Yu, G Wong, A Thompson) of the guidelines committee must be free of any conflict of interest (COI) or other biases that could undermine the integrity or credibility of the work. The Chair, Co-Chairs and all panel members with relevant $\mathrm{COI}$ were required to declare the situation and recused themselves from any relevant discussions, voting, and drafting of recommendations. The Chair and Co-Chairs were responsible for writing up the guidelines with the support of all panel members. All recommendations were graded according to the Grading of Recommendations Assessment, Development and Evaluation (GRADE) system [51]. The recommendations were presented at the 30th APASL hybrid annual meeting in Bangkok, Thailand (6th Feb 2021), with the comments incorporated.

\section{Immunopathogenesis of hepatitis due to HBV reactivation}

Hepatitis B virus (HBV) is a hepatotropic virus and after entry into hepatocytes, the HBV nucleocapsid containing partially double-stranded HBV DNA (dsDNA) enters the nucleus where the viral polymerase repairs dsDNA into fulllength, covalently closed circular (cccDNA), the nuclear reservoir of HBV. Reverse transcription, viral replication, and encapsidation occur in the cytoplasm before either viral assembly and release or recycling of the nascent nucleocapsid into the nucleus to replenish the pool of cccDNA [52]. It is the persistence of these low levels of cccDNA in hepatocytes which are thought to explain the long-term risk of HBVr with potent IST that exists even in individuals who have cleared the HBV infection, with serological clearance of hepatitis B surface antigen (HBsAg) [53]. The clinical outcome of HBV infection is highly dependent on a complex interplay between the virus-specific host immune response involving cytotoxic $\mathrm{HBV}$-specific CD8 T cell and natural killer (NK)/NK-T cell responses, cytokine-mediated non-cytolytic responses as well as B-cell-mediated humoral immunity [54]. In keeping with this, resolution of HBV infection with loss of HBsAg with or without the development of anti-HBs has been demonstrated to require CD4 helper $\mathrm{T}$ cells for the efficient development of virus-specific adaptive CD8 $\mathrm{T}$ cell responses and B-cell antibody production [55].

To date, the key biological pathways leading to the development or severity of clinically significant hepatitis due to HBVr are not well defined, other than by extrapolation from the mechanism of action of the etiological agent. There are 
also little data linking HBV sequence variation to risk or severity of HBV reactivation, and it should be assumed that all HBV genotypes and variants may be associated with reactivation. Nonetheless, based on serial measurements, HBV serological markers and liver function test, hepatitis due to HBVr have been identified as a 2-stage process. The initial phase is characterized by enhanced viral replication accompanied by markedly increase hepatic expression of viral antigen. It is postulated that this initial phase of $\mathrm{HBVr}$ occurs as a result of drug treatment that directly or indirectly inhibits the anti-HBV immune response targeted against HBV, with the highest risk associated with B cell depleting therapies [56, 57] and hematopoietic stem cell transplant (HSCT) [4-6]. HBVr has also been reported with the use of IST in solid organ transplantation, traditional chemotherapies including trans-arterial chemo-embolisation for hepatocellular carcinoma, [58] as well as the more recent tyrosine kinase inhibitors, [59] tumor necrosis factor antagonist [30-35] and proteasome inhibitor for the treatment of various malignancy and autoimmune diseases [60]. The HBV genome also contains a steroid-responsive element, and prolonged corticosteroid therapy has been associated with a moderate to high risk of HBVr [61]. HBVr has also been reported to occur indirectly in $\mathrm{HCV}$ and HDV co-infected patients as a result of antiviral therapy for $\mathrm{HCV}$, or $\mathrm{HDV}$ respectively $[62,63]$. This phenomenon reflects virus-virus interactions where the host immune response to one hepatitis virus inhibits replication of the other-normally HCV or HDV are dominant over HBV — and antiviral therapy for the dominant virus results in a secondary down-regulation of immune pathways that allow HBV replication to increase. The second phase occurred during immune reconstitution on withdrawal of the IST, [6] continuous rapid inhibition of HCV by direct-acting antiviral agents (DAAs) [40] or HIV by non-HBV active HAART therapy [64]. The immune response to the markedly enhanced hepatic expression of HBV antigen leads to liver injury, manifested as hepatitis, icteric hepatitis and fulminant acute-on-chronic hepatic failure (Fig. 1).

\section{Incidence of $\mathrm{HBVr}$ treated with immunosuppressive agents}

\section{HBsAg positive patients (Table 1)}

Data from Japan and Hong Kong have shown a 45-100\% risk of $\mathrm{HBVr}$ and $15 \%$ hepatic failure in $\mathrm{HBsAg}$ positive patients receiving HSCT without antiviral prophylaxis $[65,66]$. Two

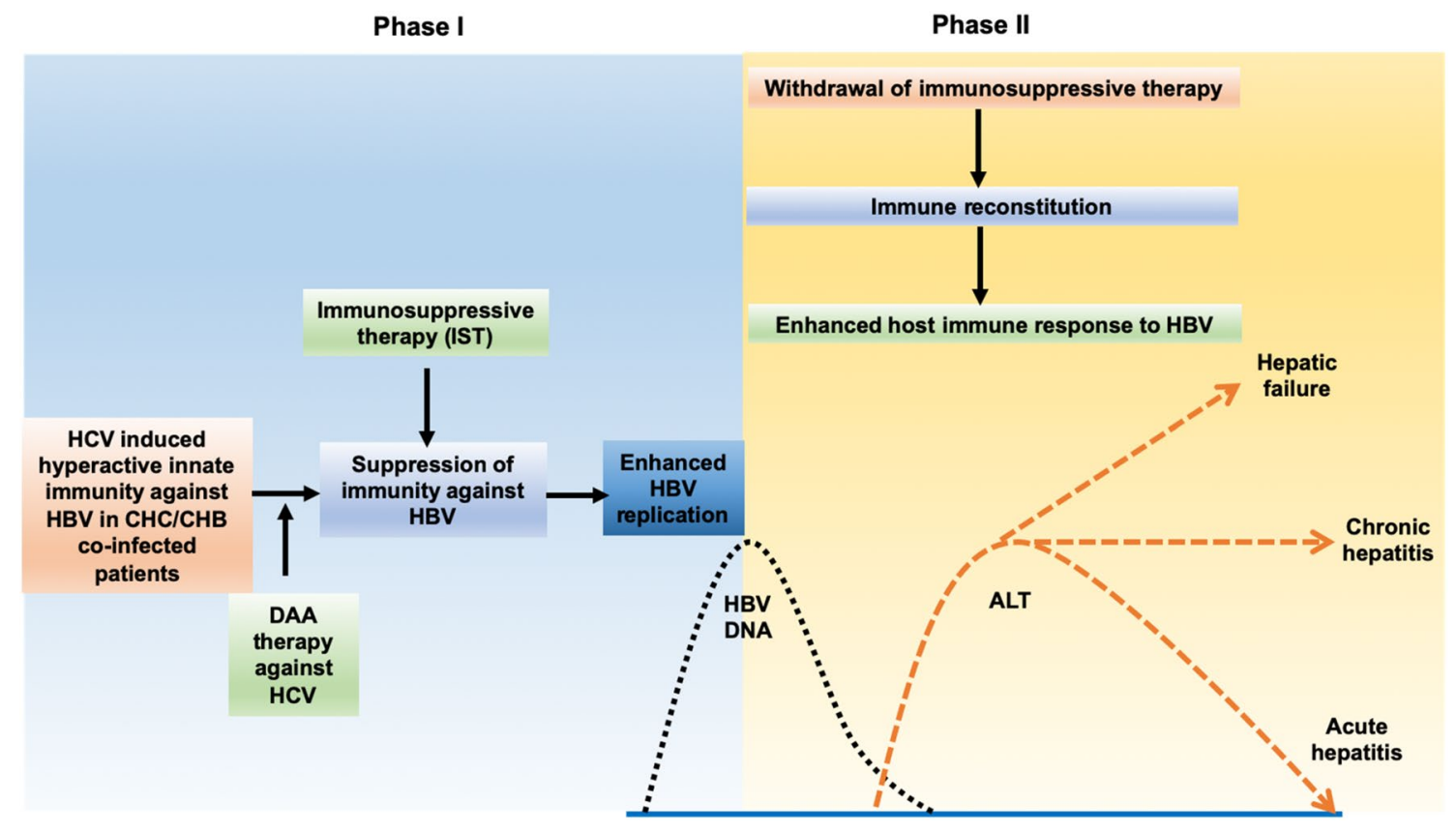

Fig. 1 Pathogenesis of hepatitis due to hepatitis $\mathrm{B}$ virological reactivation (HBVr). Hepatitis due to $\mathrm{HBVr}$ is a two-phase process with an initial phase of enhanced HBV replication and hepatocyte expression of HBV antigen due to attenuation of host immunity against HBV. The use of steroid could further augment viral replication due to its effect on steroid-responsive elements in HBV. Attenuation of host immunity against HBV replication can also be related to removal of hyperactive innate immunity with DAA therapy against co-infected
$\mathrm{HCV}$. The second phase is characterized by immune reconstitution on withdrawal of immunosuppressive effect on $\mathrm{HBVr}$ due to withdrawal of the immunosuppressive therapy or continuous rapid suppression of HCV by DAAs. This will initiate the mounting of host immune response against heavily HBV antigen-laden hepatocyte, resulting in liver injury, manifested as elevation of serum ALT with mild hepatitis, icteric hepatitis, hepatic failure or even death 
Table 1 HBV reactivation and related complications among HBsAg-positive patients without NUC prophylaxis in Asia-Pacific region

Authors (year) Country/Region Study design HBVrcase (n) Total case (n) HBVr rate HBV-related HBV-related HBV-related hepatitis (n) hepatitis rate mortality rate

Hematopoetic stem cell transplantation

\begin{tabular}{|c|c|c|c|c|c|c|c|c|}
\hline $\begin{array}{l}\text { Nakamoto } \\
\text { (2014) [65] }\end{array}$ & Japan & OB & 2 & 2 & $100 \%$ & 2 & $100 \%$ & $0 \%$ \\
\hline Lau (2002) [66] & HK & OB & 9 & 20 & $45 \%$ & 3 & $15 \%$ & NA \\
\hline \multicolumn{9}{|l|}{ Cancer diseases } \\
\hline \multicolumn{9}{|l|}{ Cytotoxic agents } \\
\hline \multicolumn{9}{|l|}{ Lymphoma } \\
\hline Lok (1991) [3] & HK & $\mathrm{OB}$ & 13 & 27 & $48 \%$ & 7 & $26 \%$ & $3.7 \%$ \\
\hline Lau (2003) [14] & HK & $\mathrm{RCT}$ & 8 & 15 & $53 \%$ & 7 & $47 \%$ & $0.0 \%$ \\
\hline \multirow{2}{*}{$\begin{array}{l}\text { Cheng (2003) } \\
\text { [70] }\end{array}$} & Taiwan & OB steroid + & 18 & 25 & $72 \%$ & 15 & $60 \%$ & $4.0 \%$ \\
\hline & & OB steroid- & 9 & 25 & $36 \%$ & 8 & $32 \%$ & $0.0 \%$ \\
\hline Hsu (2008) [16] & Taiwan & RCT CHOP & 14 & 25 & $56 \%$ & 12 & $48 \%$ & $0.0 \%$ \\
\hline Total & & & 62 & 117 & $53 \%$ & 49 & $42 \%$ & $0-4 \%$ \\
\hline \multicolumn{9}{|c|}{ Hematologic malignancies } \\
\hline $\begin{array}{l}\text { Chen (2018) } \\
\text { [71] }\end{array}$ & Taiwan & OB & 71 & 115 & $62 \%$ & NA & NA & NA \\
\hline \multicolumn{9}{|l|}{ Breast Cancer } \\
\hline Yeo (2004) [67] & HK & $\mathrm{OB}$ & 17 & 41 & $41 \%$ & NA & NA & \\
\hline $\begin{array}{l}\text { Long (2011) } \\
\text { [104] }\end{array}$ & China & $\mathrm{RCT}$ & 6 & 21 & $29 \%$ & 0 & $0.0 \%$ & $0.0 \%$ \\
\hline $\operatorname{Kim}(2007)[68]$ & Korea & $\begin{array}{l}\text { OB + anthracy- } \\
\text { cline }\end{array}$ & 23 & 111 & $21 \%$ & 23 & $21 \%$ & \\
\hline Lee (2014) [73] & Korea & $\begin{array}{l}\mathrm{OB} \pm \text { anthracy- } \\
\text { cline }\end{array}$ & 13 & 92 & $14 \%$ & 6 & $6.5 \%$ & $0.0 \%$ \\
\hline Total & & & 59 & 265 & $22 \%$ & 29 & $11 \%$ & $0-1 \%$ \\
\hline \multicolumn{9}{|c|}{ Hepatocellular carcinoma } \\
\hline \multicolumn{9}{|c|}{ TACE } \\
\hline $\begin{array}{c}\text { Jang (2006) } \\
{[74]}\end{array}$ & Korea & $\mathrm{RCT}$ & 15 & 35 & $43 \%$ & 11 & $31 \%$ & $3.0 \%$ \\
\hline $\begin{array}{l}\text { Jang (2006) } \\
{[75]}\end{array}$ & Korea & OB & 62 & 205 & $30 \%$ & 32 & $16 \%$ & $0.5 \%$ \\
\hline Total & & & 77 & 240 & $32 \%$ & 43 & $18 \%$ & $0.5-3 \%$ \\
\hline \multicolumn{9}{|c|}{ Systemic Chemotherapy } \\
\hline $\begin{array}{l}\text { Yeo (2004) } \\
\text { [72] }\end{array}$ & HK & OB & 37 & 102 & $36 \%$ & 23 & $23 \%$ & $12 \%$ \\
\hline \multicolumn{9}{|c|}{ Tyrosine kinase inhibitor } \\
\hline $\begin{array}{l}\text { Uhm (2018) } \\
\text { [23] }\end{array}$ & Korea & OB-CML & 12 & 46 & $26 \%$ & NA & NA & $0.0 \%$ \\
\hline $\begin{array}{l}\text { Wang (2019) } \\
{[24]}\end{array}$ & Taiwan & OB-CML & 5 & 13 & $38.5 \%$ & 3 & $23 \%$ & $0.0 \%$ \\
\hline $\begin{array}{l}\text { Yao (2019) } \\
\text { [25] }\end{array}$ & Taiwan & $\begin{array}{l}\text { OB-NSCLC- } \\
\text { EGFRI }\end{array}$ & 16 & 171 & $9.4 \%$ & NA & NA & NA \\
\hline Total & & & 33 & 230 & $14 \%$ & & & \\
\hline \multicolumn{9}{|c|}{ Immune checkpoint inhibitors } \\
\hline $\mathrm{Pu}(2020)[27]$ & Asia-Pacific & Review & 2 & 22 & $9.1 \%$ & 2 & $9.1 \%$ & $0 \%$ \\
\hline $\begin{array}{l}\text { Zhang (2019) } \\
\text { [28] }\end{array}$ & China & OB & 5 & 29 & $17 \%$ & 4 & $14 \%$ & $0 \%$ \\
\hline $\begin{array}{l}\text { Lee }(2020) \\
\text { [29] }\end{array}$ & Taiwan & OB & 1 & 6 & $17 \%$ & 1 & $17 \%$ & $0 \%$ \\
\hline Total & & & 8 & 57 & $14 \%$ & 7 & $11.7 \%$ & $0 \%$ \\
\hline
\end{tabular}


Table 1 (continued)

\begin{tabular}{|c|c|c|c|c|c|c|c|c|}
\hline Authors (year) & Country/Region & Study design & HBVr case $(n)$ & Total case $(n)$ & HBVr rate & $\begin{array}{l}\text { HBV-related } \\
\text { hepatitis (n) }\end{array}$ & $\begin{array}{l}\text { HBV-related } \\
\text { hepatitis rate }\end{array}$ & $\begin{array}{l}\text { HBV-related } \\
\text { mortality rate }\end{array}$ \\
\hline \multicolumn{9}{|c|}{ Rheumatic disorders } \\
\hline Lan (2011) [30] & Taiwan & OB (anti-TNF) & 5 & 8 & $63 \%$ & 5 & $63 \%$ & $0 \%$ \\
\hline $\begin{array}{l}\text { Tamori (2011) } \\
\text { [31] }\end{array}$ & Japan & OB (anti-TNF) & 2 & 5 & $40 \%$ & NA & NA & $0 \%$ \\
\hline Ryu (2012) [32] & Korea & OB (anti-TNF) & 4 & 29 & $14 \%$ & 2 & $6.9 \%$ & $0 \%$ \\
\hline Tan (2012) [33] & China & OB (c-DMARD) & 2 & 23 & $9 \%$ & 0 & $0 \%$ & $0 \%$ \\
\hline Lee (2013) [34] & Korea & Review & 14 & 74 & $19 \%$ & & NA & NA \\
\hline $\begin{array}{l}\text { Chen (2017) } \\
\text { [35] }\end{array}$ & Taiwan & OB (c-DMARD) & 30 & 123 & $24 \%$ & & NA & NA \\
\hline Total & & & 57 & 262 & $22 \%$ & 7 & $11.7 \%$ & $0 \%$ \\
\hline
\end{tabular}

meta-analyses in Asia-Pacific region have shown $>30 \%$ risk of HBVr among HBsAg-positive lymphoma patients receiving rituximab-containing regimens [56, 57]. Among HBsAgpositive breast cancers patients receiving chemotherapy in Asia-Pacific region, the risks of $\mathrm{HBVr}$ and $\mathrm{HBV}$-related hepatitis flare were around $22 \%$ (range 14-41\%) and $11 \%$ (range 0-21\%), respectively [67-69, 73]. The risk of $\mathrm{HBVr}$ among HBsAg-positive cancer patients receiving steroidcontaining regimen was $26-72 \%$, compared to $13-36 \%$ among those receiving non-steroid-containing regimens [70]. For patients with $\mathrm{HBV}$-related $\mathrm{HCC}$ receiving transarterial chemo-embolization (TACE), data from South Korea observed a risk of $32 \%(30-43 \%)$ for $\mathrm{HBVr}$ and $18 \%$ (16-31\%) for HBV-related hepatitis flare [74, 75]. Baseline HBV DNA levels > $2000 \mathrm{IU} / \mathrm{ml}$, baseline cirrhosis and history of multiple-modality therapy for $\mathrm{HCC}$ were associated with a higher risk of $\operatorname{HBVr}[72,74,75]$.

\section{Targeted therapies, monoclonal antibodies, biologics (Table 1)}

TKIs are currently widely used as target therapy for lung cancers and chronic myeloid leukemia (CML). The HBVr risk was 26-38.5\% among CML patients receiving imatinib from Taiwan and Korea studies [23, 24]. A recent Taiwanese study observed a moderate $\mathrm{HBVr}$ risk of $9.4 \%$ among patients with non-small cell lung cancers receiving epidermal growth factor receptor inhibitor [25].

ICIs are now approved for the treatment of various cancers. Case series studies from Taiwan and China showed that the risk of HBVr and its related hepatitis among HBsAgpositive cancer patients with ICIs therapy to be $14 \%$ (range 9.1-17\%) and $11.7 \%$ (range 9.1-17\%), respectively [27-29].

TNF- $\alpha$ inhibitors used for autoimmune diseases, such as rheumatic disorders and inflammatory bowel diseases, have been reported to be associated with $\mathrm{HBVr}$ risks between 14 and $63 \%$, amid a relatively small case number in the published series [30-32]. The American Gastroenterological
Association Institute guideline on the prevention and treatment of hepatitis B virus reactivation during immunosuppressive drug therapy also considered anti-TNF as moderate risk [17].

Disease-modifying antirheumatic drugs (bDMARDs/ sDMARDs) had a HBVr risk of around 22\% (range 9-63\%) among HBsAg-positive patients [31-35]. HBVr, hepatitis flare-up and even fulminant hepatic failure had been observed in HBsAg-positive rheumatic patients receiving tocilizumab, an IL-6 receptor monoclonal antibody [76, 77]. Further studies are called upon in this field as anti- IL-6 was being used in the current COVID-19 pandemic in some parts of the world [78].

\section{HBsAg (-)/anti-HBc (+) patients (Table 2)}

Data from Asia-Pacific region observed 6\%-29\% risk of $\mathrm{HBVr}$ in resolved $\mathrm{HBV}$ patients receiving HSCT without antiviral prophylaxis [65, 79-81]. A meta-analysis showed that lymphoma patients receiving rituximab-containing regimens had significantly higher risk of $\mathrm{HBVr}$ than those receiving non-rituximab-containing regimens (10\% vs $4 \%$ ) [82]. Kusumoto et al. had shown that in the phase 3 GOYA and GALLIUM studies, there was no significant difference in the risk of HBV reactivation between obinutuzumab- and rituximab-based immunochemotherapy $(p=0.17)$ [83]. A meta-analysis of 328 solid tumor patients with resolved HBV infection from 3 studies showed a median HBVr risk of 3\% (range 0.3-9\%) [69]. Recently, a study in Hong Kong showed a one-year incidence of HBsAg seroreversion of $1.8 \%$ among patients with isolated anti-HBc seropositivity receiving steroid therapy [84]. All combinations of corticosteroids dosage and duration greater than 7 days increased the risk of hepatitis flare [84].

Data of HBVr risk among HBsAg (-)/anti-HBc (+) patients receiving TKI or ICI are limited. A recent Taiwanese study observed that none of $123 \mathrm{CML}$ patients with resolved HBV receiving TKI experienced HBVr [24]. 
Table 2 HBV reactivation and related complications among HBsAg-negative patients without pre-emptive NUCs

\begin{tabular}{|c|c|c|c|c|c|c|c|c|}
\hline Authors (year) & Country/Region & Study design & $\operatorname{HBVr}$ case $(n)$ & Total case $(n)$ & HBVr rate & $\begin{array}{l}\text { HBV-related } \\
\text { hepatitis }(n)\end{array}$ & $\begin{array}{l}\text { HBV-related } \\
\text { hepatitis rate }\end{array}$ & $\begin{array}{l}\text { HBV-related } \\
\text { mortality rate }\end{array}$ \\
\hline \multicolumn{9}{|c|}{ Hematopoetic stem cell transplantation } \\
\hline $\begin{array}{l}\text { Nakamoto } \\
\text { (2014) [65] }\end{array}$ & Japan & OB & 6 & 83 & $7 \%$ & NA & NA & NA \\
\hline Seto (2017) [79] & HK & $\mathrm{OB}$ & 13 & 62 & $21 \%$ & NA & NA & NA \\
\hline Wu (2020) [80] & China & OB & 25 & 441 & $6 \%$ & NA & NA & NA \\
\hline \multirow{2}{*}{$\begin{array}{l}\text { Nishikawa } \\
\text { (2020) [81] }\end{array}$} & Japan & $\mathrm{OB}$ & 13 & 67 & $19 \%$ & NA & NA & NA \\
\hline & Total & & 57 & 653 & $8.7 \%$ & & & \\
\hline \multicolumn{9}{|c|}{ Lymphoma-anti-CD20-containing C/T } \\
\hline Yeo (2009) [8] & HK & $\mathrm{RCT}$ & 5 & 21 & $24 \%$ & 5 & $24 \%$ & $4 \%$ \\
\hline $\begin{array}{l}\text { Matsue (2010) } \\
\text { [9] }\end{array}$ & Japan & OB & 5 & 56 & $9 \%$ & 5 & $8.9 \%$ & $0 \%$ \\
\hline $\begin{array}{c}\text { Koo (2011) } \\
{[105]}\end{array}$ & Singapore & OB & 2 & 62 & $3 \%$ & 2 & $3.2 \%$ & $1.6 \%$ \\
\hline $\begin{array}{l}\text { Huang (2013) } \\
{[10]}\end{array}$ & Taiwan & RCT & 7 & 39 & $18 \%$ & 2 & $5.1 \%$ & $0 \%$ \\
\hline Seto (2014) [11] & HK & OB & 19 & 63 & $30 \%$ & 0 & $0.0 \%$ & $0 \%$ \\
\hline Hsu (2014) [12] & Taiwan & OB & 27 & 143 & $19 \%$ & 10 & $7.0 \%$ & $0 \%$ \\
\hline $\begin{array}{l}\text { Kusumoto } \\
\text { (2019) [83] }\end{array}$ & $\begin{array}{c}\text { Asia Pacific, } \\
\text { Europe, } \\
\text { Canada }\end{array}$ & OB & 25 & 232 & 10.8 & NA & NA & NA \\
\hline & Total & & 65 & 384 & $16.9 \%$ & 24 & $6 \%$ & \\
\hline \multicolumn{9}{|c|}{ Lymphoma-non-rituximab $\mathrm{C} / \mathrm{T}$} \\
\hline Lok (1991) [3] & HK & OB & 2 & 45 & $4 \%$ & 2 & $4.4 \%$ & $0 \%$ \\
\hline \multirow[t]{2}{*}{ Yeo (2009) [8] } & HK & RCT & 0 & 25 & $0 \%$ & 0 & $0.0 \%$ & $0 \%$ \\
\hline & Total & & 2 & 70 & $3 \%$ & 2 & $3 \%$ & \\
\hline \multicolumn{9}{|c|}{ Hematologic malignancies } \\
\hline $\begin{array}{l}\text { Chen (2018) } \\
\text { [71] }\end{array}$ & Taiwan & $\begin{array}{l}\text { OB (585 anti- } \\
\text { HBc }[+])\end{array}$ & 41 & 1676 & $2.4 \%$ & 36 & $2.1 \%$ & $0.06 \%$ \\
\hline \multicolumn{9}{|l|}{ TKI } \\
\hline $\begin{array}{l}\text { Wang (2019) } \\
\text { [24] }\end{array}$ & Taiwan & $\begin{array}{c}\text { OB-CML (55\% } \\
\text { anti-HBc+) }\end{array}$ & 0 & 123 & $0.0 \%$ & 0 & $0 \%$ & $0.0 \%$ \\
\hline \multicolumn{9}{|l|}{ Rheumatic disorders } \\
\hline Lan (2011) [30] & Taiwan & OB (anti-TNF) & 1 & 70 & $1.4 \%$ & 1 & $1.4 \%$ & $0 \%$ \\
\hline $\begin{array}{l}\text { Tamori (2011) } \\
\text { [31] }\end{array}$ & Japan & OB (anti-TNF) & 1 & 45 & $2.2 \%$ & 1 & $2.2 \%$ & $0 \%$ \\
\hline $\operatorname{Tan}(2012)$ [33] & China & OB (c-DMARD) & 2 & 188 & $1.1 \%$ & 1 & $0.5 \%$ & $0 \%$ \\
\hline $\begin{array}{l}\text { Mori (2011) } \\
{[106]}\end{array}$ & Japan & OB (anti-TNF) & 2 & 60 & $3.3 \%$ & 0 & $0.0 \%$ & $0 \%$ \\
\hline $\begin{array}{l}\text { Urata (2011) } \\
\text { [107] }\end{array}$ & Japan & OB (anti-TNF) & 7 & 135 & $5.2 \%$ & NA & NA & $0 \%$ \\
\hline \multirow{2}{*}{$\begin{array}{l}\text { Watanabe } \\
\text { (2019) [108] }\end{array}$} & Japan & OB (c-DMARD) & 7 & 152 & $4.6 \%$ & & & \\
\hline & Total & & 20 & 650 & $3.1 \%$ & 3 & $0.8 \%$ & \\
\hline
\end{tabular}

Relating to TNF- $\alpha$ inhibitors and DAMARDs, data from Asia-Pacific regions demonstrated a HBVr risk of around $3.1 \%$ (range $1.1-5.2 \%$ ) [30-32]. A recent Taiwanese study reported one $(1.6 \%)$ out of 64 rheumatic patients receiving tocilizumab experienced HBVr [76]. Further studies are needed in these areas.

\section{$\mathrm{HBVr}$ among patients with $\mathrm{HBV}$ and $\mathrm{HCV}$ co-infections} (Table 3)

The recent advance of DAAs has dramatically improved the treatment success of $\mathrm{CHC}$ infection, making $\mathrm{HCV}$ 
Table 3 Hepatitis B virus reactivation (HBVr) among patients with HBV and hepatitis C virus (HCV) co-infection or HCV infection with resolved HBV infection after direct-acting antiviral (DAA) treatment in Asia-Pacific region

\begin{tabular}{|c|c|c|c|c|c|c|c|c|c|}
\hline $\begin{array}{l}\text { Authors } \\
\text { (year) }\end{array}$ & $\begin{array}{l}\text { Country/ } \\
\text { Region }\end{array}$ & $\begin{array}{l}\text { Total } \\
\text { patients }(n)\end{array}$ & $\begin{array}{l}\text { Observa- } \\
\text { tion periods } \\
\text { (months } \\
\text { post-EOT) }\end{array}$ & $\begin{array}{l}\text { Patients } \\
\text { with HBVr } \\
{[n(\%)]}\end{array}$ & $\begin{array}{l}\text { Patients } \\
\text { with HBVr } \\
\text { and hepati- } \\
\text { tis }[n(\%)]\end{array}$ & $\begin{array}{l}\text { Patients } \\
\text { with HBVr } \\
\text { and icteric } \\
\text { hepatitis [ } n \\
(\%)]\end{array}$ & $\begin{array}{l}\text { Mortality } \\
\text { in patients } \\
\text { with } \mathrm{HBVr} \\
{[n(\%)]}\end{array}$ & $\begin{array}{l}\text { Pre-DAA } \\
\text { HBV DNA } \\
(-) \text { in } \\
\text { patients } \\
\text { with HBVr } \\
{[n(\%)]}\end{array}$ & Predictors \\
\hline \multicolumn{10}{|c|}{ HBsAg-positive HBV/HCV co-infected patients } \\
\hline $\begin{array}{l}\text { Gane } \\
(2016) \\
{[37]}\end{array}$ & $\begin{array}{l}\text { New Zea- } \\
\text { land }\end{array}$ & 8 & 3 & $6(38)$ & $0 / 8(0)$ & $0 / 8(0)$ & $0 / 6(0)$ & $3 / 6(50)$ & NA \\
\hline $\begin{array}{l}\text { Doi } \\
\qquad(2017) \\
{[38]}\end{array}$ & Japan & 4 & 3 & $2(50)$ & $0 / 4(0)$ & $0 / 4(0)$ & $0 / 2(0)$ & $2 / 2(100)$ & NA \\
\hline $\begin{array}{l}\text { Kawagishi } \\
(2017) \\
{[39]}\end{array}$ & Japan & 1 & 3 & $1(100)$ & $0 / 1(0)$ & $0 / 1(0)$ & $0 / 1(0)$ & $1 / 1(100)$ & NA \\
\hline $\begin{array}{l}\text { Wang } \\
\qquad \begin{array}{l}(2017) \\
{[40]}\end{array}\end{array}$ & China & 10 & 3 & $3(30)$ & $3 / 10(30)$ & $1 / 10(10)$ & $0 / 3(0)$ & NA & NA \\
\hline $\begin{array}{l}\text { Tamori } \\
(2018) \\
{[41]}\end{array}$ & Japan & $22 *$ & 3 & $3(14)$ & $2 / 22(9)$ & $0 / 0(0)$ & $0 / 3(0)$ & $3 / 3(100)$ & NA \\
\hline $\begin{array}{l}\text { Liu (2018) } \\
\text { [42] }\end{array}$ & Taiwan & 111 & 3 & $50(45)$ & $5 / 111(5)$ & $1 / 111(1)$ & $0 / 50(0)$ & $11 / 50(22)$ & NA \\
\hline $\begin{array}{l}\text { Liu (2017) } \\
\text { [44] }\end{array}$ & Taiwan & 12 & 3 & $2(17)$ & $0 / 12(0)$ & $0 / 12(0)$ & $0 / 2(0)$ & $1 / 2(50)$ & NA \\
\hline $\begin{array}{l}\text { Lee } \\
\qquad(2018) \\
{[45]}\end{array}$ & Taiwan & 7 & 3 & $2(29)$ & $1 / 7$ (14) & $0 / 7(0)$ & $0 / 2(0)$ & $1 / 2(50)$ & NA \\
\hline $\begin{array}{l}\text { Yeh } \\
\quad(2020) \\
{[46]}\end{array}$ & Taiwan & $66^{*}$ & $\begin{array}{l}3-36 \text { (mean } \\
11)\end{array}$ & $30(45)$ & $6 / 66(9)$ & $3 / 66(5)$ & $2 / 30(7)^{* *}$ & $15 / 30(50)$ & $\begin{array}{l}\text { BL ALT } \geq 80 \mathrm{U} / \mathrm{L} ; \\
\mathrm{HBsAg}>10 \mathrm{IU} / \\
\mathrm{ml}\end{array}$ \\
\hline Total & & 241 & & 99 (41.1) & $17(7.1)$ & $5(2.1)$ & $2 / 99(2.0)$ & $\begin{array}{l}37 / 96 \\
\quad(38.5)\end{array}$ & \\
\hline \multicolumn{10}{|c|}{ HBsAg-negative patients positive for anti-HBc antibody and/or anti-HBs antibody } \\
\hline $\begin{array}{l}\text { Doi } \\
\qquad(2017) \\
{[38]}\end{array}$ & Japan & 155 & 3 & $3(1.9)$ & $0 / 155(0)$ & $0 / 155(0)$ & $0 / 3(0)$ & $3 / 3(100)$ & $\begin{array}{l}\text { BL High ALT; } \\
\text { Low Anti-HBs } \\
\text { titer }^{+}\end{array}$ \\
\hline $\begin{array}{l}\text { Kawagishi } \\
\text { (2017) } \\
{[39]}\end{array}$ & Japan & 84 & 1 & $4(2.6)$ & $1 / 84(1)$ & $0 / 84(0)$ & $0 / 4(0)$ & $4 / 4(100)$ & $\begin{array}{l}\text { BL Anti-HBs (-) } \\
\text { or }<30 \mathrm{mIU} / \mathrm{ml} \text {; } \\
\text { EOT Anti-HBs } \\
(-) \text { or }<12 \mathrm{mIU} / \\
\mathrm{ml}^{\ddagger}\end{array}$ \\
\hline $\begin{array}{l}\text { Yeh } \\
\quad(2017) \\
{[43]}\end{array}$ & Taiwan & 57 & 3 & $0(0)$ & $0 / 57(0)$ & $0 / 57(0)$ & $0(0)$ & NA & NA \\
\hline $\begin{array}{l}\text { Sulkowski } \\
(2016) \\
{[47]}\end{array}$ & $\begin{array}{r}\text { Taiwan/ } \\
\text { Korea }\end{array}$ & 103 & 3 & $0(0)$ & $0 / 103(0)$ & $0 / 103(0)$ & $0(0)$ & NA & NA \\
\hline $\begin{array}{l}\text { Wang } \\
\qquad(2017) \\
{[40]}\end{array}$ & China & 124 & 3 & $0(0)$ & $0 / 124(0)$ & $0 / 124(0)$ & $0(0)$ & NA & NA \\
\hline $\begin{array}{l}\text { Tamori } \\
(2018) \\
{[41]}\end{array}$ & Japan & 765 & 3 & $1(0.1)$ & $0 / 765(0)$ & $0 / 765(0)$ & $0(0)$ & $1 / 1(100)$ & NA \\
\hline
\end{tabular}


Table 3 (continued)

\begin{tabular}{|c|c|c|c|c|c|c|c|c|c|}
\hline $\begin{array}{l}\text { Authors } \\
\text { (year) }\end{array}$ & $\begin{array}{l}\text { Country/ } \\
\text { Region }\end{array}$ & $\begin{array}{l}\text { Total } \\
\text { patients }(n)\end{array}$ & $\begin{array}{l}\text { Observa- } \\
\text { tion periods } \\
\text { (months } \\
\text { post-EOT) }\end{array}$ & $\begin{array}{l}\text { Patients } \\
\text { with HBVr } \\
{[n(\%)]}\end{array}$ & $\begin{array}{l}\text { Patients } \\
\text { with HBVr } \\
\text { and hepati- } \\
\text { tis }[n(\%)]\end{array}$ & $\begin{array}{l}\text { Patients } \\
\text { with HBVr } \\
\text { and icteric } \\
\text { hepatitis [ } n \\
(\%)]\end{array}$ & $\begin{array}{l}\text { Mortality } \\
\text { in patients } \\
\text { with } \mathrm{HBVr} \\
{[n(\%)]}\end{array}$ & $\begin{array}{l}\text { Pre-DAA } \\
\text { HBV DNA } \\
(-) \text { in } \\
\text { patients } \\
\text { with HBVr } \\
{[n(\%)]}\end{array}$ & Predictors \\
\hline $\begin{array}{c}\text { Ogawa } \\
(2018) \\
{[48]}\end{array}$ & Japan & 63 & 3 & $1(2)$ & $0 / 63(0)$ & $0 / 63(0)$ & 0/1 (0) & 1/1 (100) & NA \\
\hline $\begin{array}{c}\text { Liu (2017) } \\
\text { [44] }\end{array}$ & Taiwan & 81 & 3 & $0(0)$ & $0 / 81(0)$ & $0 / 81(0)$ & $0(0)$ & NA & NA \\
\hline $\begin{array}{l}\text { Lee } \\
\qquad(2018) \\
{[45]}\end{array}$ & Taiwan & 53 & 3 & 0 & (0) & $0 / 53(0)$ & $0(0)$ & NA & NA \\
\hline Total & & 1485 & 3 & $9(0.6)$ & $1(0.07)$ & $0(0)$ & $0(0)$ & 9/9 (100) & \\
\hline
\end{tabular}

HBVr, HBV DNA increases greater than $1 \log 10 \mathrm{IU} / \mathrm{ml}$ or HBV DNA reappearance

$H B s A g$ hepatitis B surface antigen, anti-HBc anti-hepatitis B core antibody, anti-HBs anti-hepatitis B surface antibody, EOT end of treatment, $n$ number

*Excluding patients with concomitant anti-HBV NUC therapy at initiation of DAA therapy

**Both patients had cirrhosis at baseline

${ }^{\ddagger}$ Subjects of both positive and negative HBsAg analyzed together

elimination possible in the near future [85]. The successful $\mathrm{HCV}$ eradication rate with DAAs is comparable between $\mathrm{HCV}$ mono-infected and HBV/HCV co- patients. However, coinfected patients are at risk of $\mathrm{HBVr}$ during and after DAA therapy and this occurs earlier and is clinically more significant than HBVr occurring with interferon-based therapy [37-47, 86-88]. Data from Asia-Pacific region demonstrated a risk of $41.1 \%$ (range $14-100 \%$ ) for $\mathrm{HBVr}$, $7.1 \%$ (range 0-30\%) for HBV-related hepatitis flare, 2.1\% (range 0-10\%) for HBV-related icteric hepatitis, and 2\% (range 0-7\%) mortality (Table 3). The risk of HBVr was not associated with baseline HBV DNA levels. Patients with undetectable HBV DNA at baseline are still at risk of HBVr. In a recent study, baseline quantitative $\mathrm{HBsAg}$ (qHBsAg) titers were associated with HBVr. The 1-year cumulative incidence rate of $\mathrm{HBVr}$ was $42.5 \%$ in patients with baseline $\mathrm{qHBsAg}>10 \mathrm{IU} / \mathrm{ml}$, compared to $18.5 \%$ in those with baseline qHBsAg $<10 \mathrm{IU} / \mathrm{ml}$ [46].

\section{Risk stratification (Table 4)}

There are three key risk factors associated with $\mathrm{HBVr}$, [21, 89] namely: (1) Host factors: male sex, older age, presence of cirrhosis, and type of disease treated with IST, such as bone marrow transplant or solid-organ transplantation; (2) HBV virologic factors: HBsAg seropositivity, high baseline HBV DNA levels, HBeAg seropositivity, and absence of anti-HBs among patients with resolved
HBV infection, or co-infection with HCV, HDV, or HIV; (3) Type and degree of IST: B-cell-depleting therapies, such as rituximab and ofatumumab, anthracycline derivatives, such as doxorubicin and epirubicin, medium (10-20 mg/day) or high dose ( $\geq 20 \mathrm{mg} /$ day) prednisone therapy for $\geq 4$ weeks, steroid-containing chemotherapy, and TNF- $\alpha$ inhibitors, such as infliximab and etanercept.

A risk gradient for $\mathrm{HBVr}$ exists between people who are $\mathrm{HBsAg}$-positive and people who are $\mathrm{HBsAg-negative}$ but anti-HBs positive [89-91]. The risk of $\mathrm{HBVr}$ is 5 to 8 times higher among those patients who are HBsAgpositive as compared to those who were HBsAg negative but anti-HBc positive. Among patients who are HBsAgpositive the best predictor of reactivation has been shown to be the level of HBV DNA at baseline [85]. The risk of $\mathrm{HBVr}$ in patients who are $\mathrm{HBsAg}$ negative and anti-HBc positive is lower, where high risk has been reported for patients being treated with $\mathrm{B}$ cell therapies or undergoing HSCT. In individuals who are HBsAg-negative and anti$\mathrm{HBc}$ positive, the presence and titer of anti-HBs antibodies have been associated with some protection against $\mathrm{HBVr}$ $[82,92]$. However data are limited, and at present, there is insufficient evidence to support the use of anti-HBs titres for clinical decision-making in this situation.

Based on the type and duration of IST and the status of HBV infection, the risk of HBVr was established to be low $(<1 \%)$, moderate $(1-10 \%)$ and high $(>10 \%)$ (Table 4$)$ $[21,90]$. 
Table 4 Risk stratification of HBV reactivation among HBsAg-positive patients and HBsAg-negative/anti-HBc-positive patients

\begin{tabular}{|c|c|c|}
\hline \multirow[t]{2}{*}{ Risk level } & \multicolumn{2}{|l|}{ HBV serology } \\
\hline & $\operatorname{HBsAg}(+)$ & HBsAg(-)/anti-HBc(+) \\
\hline High $(>10 \%)$ & $\begin{array}{l}\text { Anti-CD20 monoclonal antibodies: Rituximab, } \\
\text { Ofatumumab, Obinutuzumab } \\
\text { Steroid (high dose) } \geq 20 \mathrm{mg} / \text { day for } \geq 4 \text { weeks } \\
\text { Anti-TNF agents with higher potency: Adali- } \\
\text { mumab, Infliximab, Golimumab, Certoli- } \\
\text { zumab } \\
\text { Anthracyclines } \\
\text { Hematopoietic stem cell transplantation (both } \\
\text { allogeneic and autologous) } \\
\text { DAA for HBV/HCV coinfection (high risk in } \\
\text { meta-analysis and prospective study), except } \\
\text { non-cirrhotics with HBsAg < } 10 \text { IU/ml } \\
\text { Immune Checkpoint inhibitors (moderate to } \\
\text { high risk): } \\
\text { Anti-PD-1: nivolumab, pembrolizumab } \\
\text { Anti-PD-L1: atezolizumab } \\
\text { Anti-CTLA-4: ipilimumab } \\
\text { Tyrosine kinase inhibitors (moderate-to-high): } \\
\text { Imatinib, Nilotinib, Dasatinib, Erlotinib, } \\
\text { Gefitinib, Osimertinib, Afatinib }\end{array}$ & $\begin{array}{l}\text { Anti-CD20 monoclonal antibodies: Rituximab, } \\
\text { Ofatumumab, Obinutuzumab } \\
\text { Allogeneic hematopoietic stem cell transplanta- } \\
\text { tion }\end{array}$ \\
\hline Moderate (1-10\%) & $\begin{array}{l}\text { Cytotoxic chemotherapy (except anthracy- } \\
\text { clines) } \\
\text { Anti-TNF agents with lower potency: Etaner- } \\
\text { cept } \\
\text { Steroid (median dose): } 10-20 \mathrm{mg} / \text { day } \\
\text { for } \geq 4 \text { weeks } \\
\text { Proteasome inhibitor: Bortezomib Usteki- } \\
\text { numab }\end{array}$ & $\begin{array}{l}\text { Anthracyclines } \\
\text { Autologous hematopoietic stem cell transplanta- } \\
\text { tion } \\
\text { Anti-TNF agents with higher potency: Adali- } \\
\text { mumab, Infliximab, Golimumab, Certolizumab } \\
\text { Proteasome inhibitor: Bortezomib Ustekinumab }\end{array}$ \\
\hline Low $(<1 \%)$ & $\begin{array}{l}\text { Methotrexate } \\
\text { Azathioprine } \\
\text { Steroid (low dose }<10 \mathrm{mg} / \text { day) } \\
\text { DAA for } \mathrm{HBV} / \mathrm{HCV} \text { coinfection for non-cir- } \\
\text { rhotic patients with } \mathrm{HBs} \mathrm{Ag}<10 \mathrm{IU} / \mathrm{ml}\end{array}$ & $\begin{array}{l}\text { Cytotoxic chemotherapy (except anthracyclines) } \\
\text { Steroid (high dose) } \geq 20 \mathrm{mg} / \text { day } \\
\text { Anti-TNF agents with lower potency: Etanercept } \\
\text { Tyrosine kinase inhibitors Imatinib, Nilotinib, } \\
\text { Dasatinib } \\
\text { DAA for HCV }\end{array}$ \\
\hline $\begin{array}{l}\text { Uncertain (More studies needed, no } \\
\text { prophylaxis recommendation until further } \\
\text { evidence) }\end{array}$ & $\begin{array}{l}\text { Abatacept } \\
\text { Tocilizumab } \\
\text { Ibrutinib } \\
\text { Alemtuzumab } \\
\text { Natalizumab } \\
\text { Ocrelizumab } \\
\text { Ibritumomab }\end{array}$ & $\begin{array}{l}\text { Immune Checkpoint inhibitors } \\
\text { Anti-PD-1: nivolumab, pembrolizumab } \\
\text { Anti-PD-L1: atezolizumab } \\
\text { Anti-CTLA-4: ipilimumab }\end{array}$ \\
\hline
\end{tabular}

\section{The rationale of pre-emptive use of nucleos(t)ide analogues}

Soon after the global registration of lamivudine, the first randomized controlled trial to compare "early" pre-emptive (start lamivudine before or at the initiation of cytotoxic chemotherapy for lymphoma) to "deferred" treatment (lamivudine initiated only when $\mathrm{HBVr}$ was detected on monitoring) was conducted in Chinese with lymphoma. The rationale was based on the hypothesis that if one can inhibit the enhanced HBV replication during the initial phase of intense IST, then on immune-reconstitution during withdrawal of the IST, the amount of HBV antigen-laden hepatocytes as target for host immunity and hence the incidence of liver injury should be drastically reduced. This will be in contrast to the "deferred" approach as the immune response to HBV-antigen laden hepatocytes has already been initiated and indeed the markedly enhanced HBV replication has already been abating. In keeping with this, "early" pre-emptive approach is found to be superior to "deferred" use of lamivudine resulting in a marked reduction in the incidence of $\mathrm{HBVr}$ and hepatitis in $\mathrm{HBsAg}$ positive with lymphoma treated with intense cytotoxic IST [14]. Similarly, in a prospective, open-label cohort study on Chinese adults of HBV inactive carriers with concurrent $\operatorname{IgAN}$ (proteinuria $\geq 3.5 \mathrm{~g} /$ day), this pre-emptive "early" use of lamivudine was found to be 
highly effective in preventing $\mathrm{HBVr}$ and its related hepatitis [93]. Subsequent systemic review and meta-analysis, based on 14 studies showed that the relative risk for both $\mathrm{HBVr}$ and HBV-related hepatitis ranged from 0.00 to 0.21 , favoring preemptive use of lamivudine. A significantly higher proportion of participants not treated with pre-emptive lamivudine suffered from a disruption of chemotherapy [94]. However, due to the low-resistant barrier of lamivudine, some patients might develop YMDD mutation and deter the effectiveness of such a pre-emptive approach. Hence, lamivudine was later replaced by high resistant barrier NUCs with adefovir, entecavir and tenofovir $[13,95,96]$. With a higher safety profile with long-term use of entecavir, tenofovir and lately TAF, their pre-emptive use to prevent $\mathrm{HBVr}$ in patients planned for IS therapy have been validated in randomized control trials (Table 5).

\section{Assessment by hepatologists for termination of NUC treatment}

All patients who are planned for IST should have HBsAg, anti-HBs and anti-HBc tested at baseline (Fig. 2). The risk of IST for HBVr should be assessed (Table 4). For HBsAgpositive patients, serum HBV DNA, and possibly qHBsAg should be checked and monitored. Current data on other biomarkers, such as anti-HBs and anti-HBc titres, HBV corerelated antigen (HBcrAg), ultra-sensitive $\mathrm{HBsAg}$ evaluation and HBV RNA, in the diagnosis and monitoring of $\mathrm{HBV}$ reactivation over the course of immunosuppressive treatments are not sufficient to be of any added practical clinical use [97]. Assessment of liver fibrosis, either invasively or non-invasively, should then be performed under the guidance of a hepatologist. All HBV treatment guidelines recommend patients with significant fibrosis ( $\mathrm{F} 2$ or greater) should receive NUCs treatment, while close monitoring may be indicated for patients without significant fibrosis. Therefore, using liver fibrosis assessment to stratify preventive therapy in low to moderate risk patients is a logical approach. Various non-invasive assessments have been developed and adopted in some international management guidelines [98, 99]. Liver stiffness measurement (LSM) with transient elastography is most widely validated and is an accurate and reproducible method to predict advanced fibrosis or cirrhosis $(\geq F 3)$ in CHB patients $[98,99]$. The key challenge of this tool is the confounding effect of alanine aminotransferase (ALT) level, such that decrease in LSM may only reflect ALT normalization. In HBVr patients, LSM should therefore be assessed after normalization of ALT levels to accurately diagnose the degree of fibrosis [98]. However, Jia et al. showed in a large cohort of Chinese patients with CHB, ALT levels up to five times the ULN did not significantly affect the diagnostic power of LSM [100]. Li et al. showed that patients with mildly elevated ALT levels had higher
LSM cut-off values than patients with normal ALT levels on predicting F2-F4 (6.5 vs $6 \mathrm{kPa})$ and F4 (10.2 vs $7.8 \mathrm{kPa})$ [101]. Using cut-offs regardless of ALT levels, the diagnostic accuracy of LSM was $81 \%$ for F2-F4, and $89 \%$ for F4. Applying ALT-stratified cut-off values, the diagnostic accuracy of LSM was $82 \%$ for predicting F2-F4, and $86 \%$ for predicting F4 [101]. In regions where transient elastography is not readily accessible, serum test formulae based on common laboratory parameters have the advantages of high applicability. Examples include aspartate aminotransferase (AST) to platelet ratio index (APRI), Forns index and Fibrosis-4 (FIB-4) score [102].

Both HBsAg positive and HBsAg negative but anti$\mathrm{HBc}$ positive patients treated with IST considered high risk should be initiated pre-emptive high-resistant barrier NUCs. For those with moderate risk, all HBsAg positive and those HBsAg negative but anti-HBc positive patients with advanced liver fibrosis or cirrhosis should be initiated preemptive high-resistant barrier NUCs. The preferred NUCs are entecavir, tenofovir or TAF. For those HBsAg negative and anti-HBc positive patients without advance fibrosis or cirrhosis, serum ALT should be monitored every 3 months. If elevated ALT $>2 \times$ baseline detected at monitoring, HBsAg and HBV DNA should be performed and high-resistant barrier NUCs initiated if either test positive. For those with low-risk, pre-emptive NUCs should be initiated in both $\mathrm{HBsAg}$ positive and $\mathrm{HBsAg}$ negative but anti-HBc positive with advanced fibrosis or cirrhosis. Serum ALT should be monitored every 3 months in both HBsAg positive and HBsAg negative but anti-HBc positive patients with lowrisk. The AASLD, AGA and EASL recommend antiviral treatment should be continued for at least 6 months after discontinuation of IST and at least 12 months for B celldepleting agents. $[17,19,20]$ Our panel recommended that under the guidance of a hepatologist, termination of NUCs would be considered 6 months after the completion of IST for $\mathrm{HBs} A g$ positive patients, without advanced liver fibrosis or cirrhosis and with low level of HBV DNA (<2000 IU/ $\mathrm{ml}$ ) before initiation of NUCs. For those who remain $\mathrm{HBsAg}$ negative but anti-HBc positive, termination of NUCs should be considered 6 months after the completion of IST. HBV DNA monitoring-guided preemptive NUCs are effective for preventing HBV-related hepatitis in these patients [83]. Close monitoring every 3 months and prompt initiation of NUCs may be more cost-effective. In the future with more solid evidence, new biomarkers, such as HBV RNA, HBcrAg, may be helpful to decide when to terminate NUCs after completion of IST [103].

\section{Comparison to previous guidelines}

In the Asian-Pacific clinical practice guidelines on the management of hepatitis B: a 2015 update, a section "Antiviral 


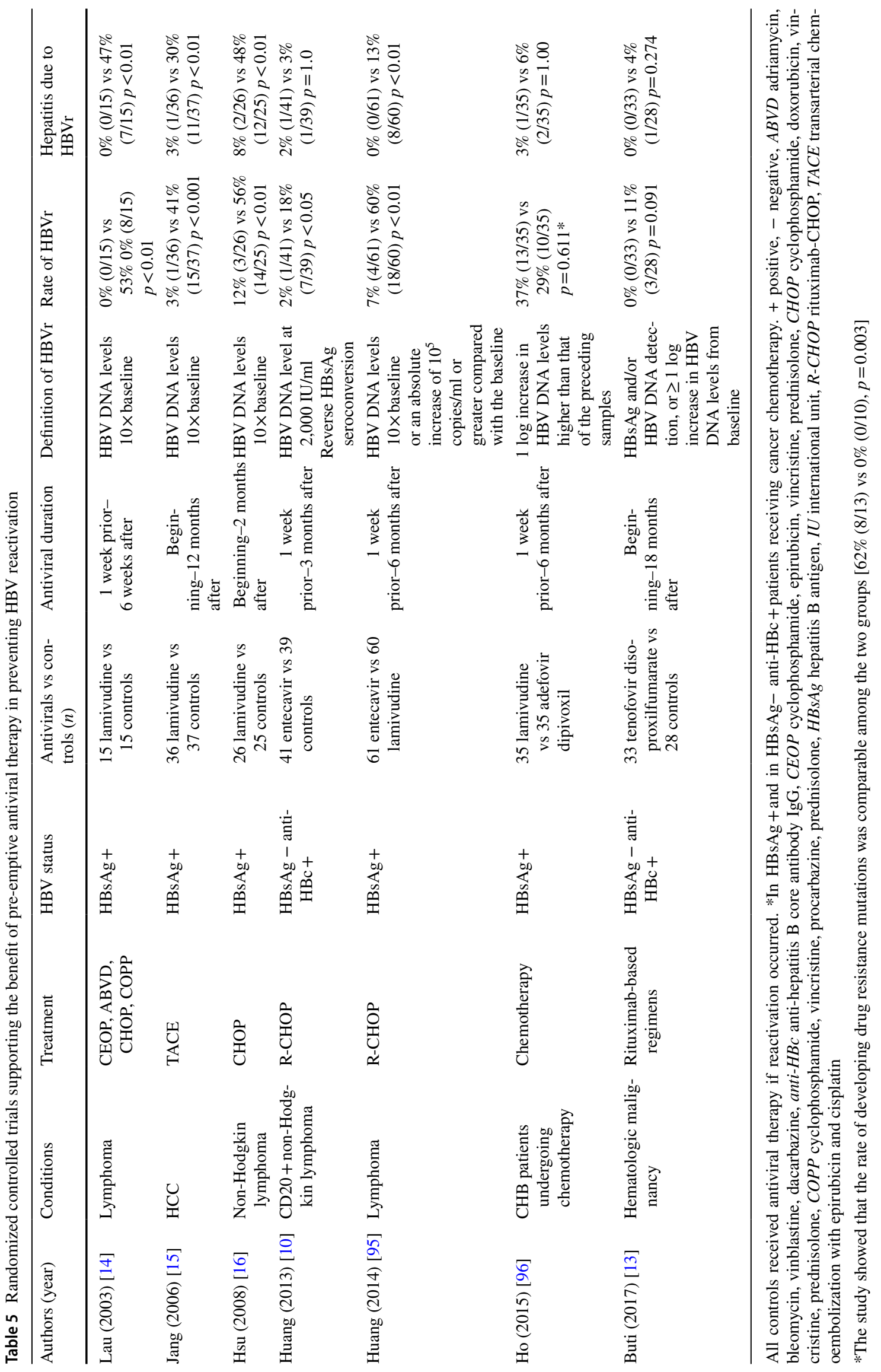




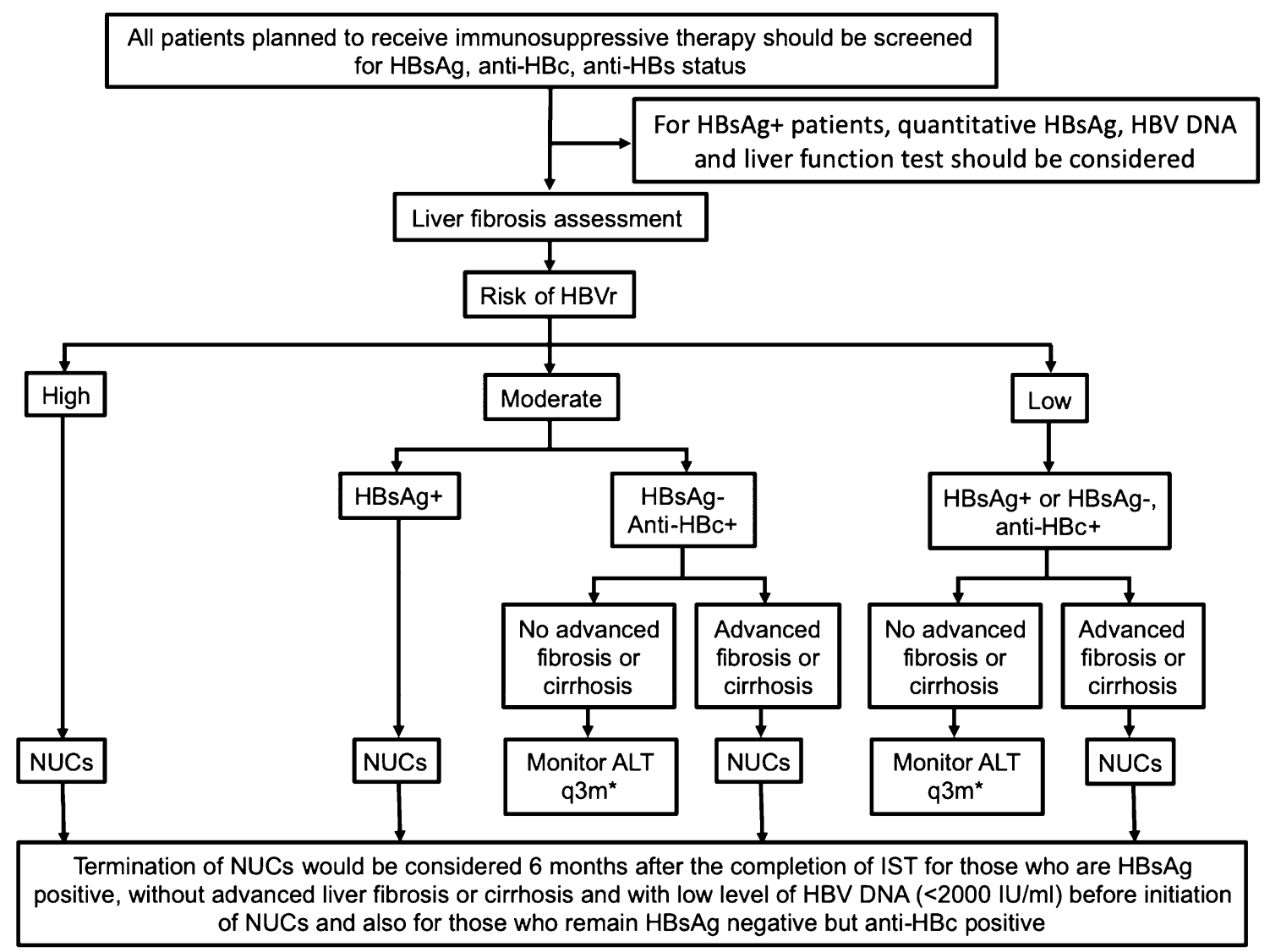

Fig. 2 Algorithm for the management of hepatitis B reactivation. All high-risk patients and moderate risk $\mathrm{HBsAg}+$ patients should be treated with pre-emptive NUCs irrespective of fibrosis status. All patients with advanced fibrosis or cirrhosis should be treated with NUCs irrespective risk stratifications. All HBsAg + patients should be treated with NUCs except for low-risk patients without advanced

prophylaxis before immunosuppressive therapy or chemotherapy" was included to discuss this important topic [18]. Over the last few years, more data have been made available to allow better stratification of the risk of $\mathrm{HBVr}$ with various immunosuppressive agents, namely new biologics, targeted therapies, immunotherapies and anti-HCV directacting antiviral agents. The recommendations remain the same for HBsAg-positive patients to whom pre-emptive NUC therapy should be started. The main changes lay on the HBsAg-negative but anti-HBc positive patients, as in the 2015 guidelines it was mentioned that further studies are needed to compare the efficacy and cost-effectiveness of different preventive strategies (pre-emptive NUCs versus monitoring). Furthermore, HBsAg-negative, anti-HBc positive patients with undetectable serum HBV DNA and who receive IST regardless of anti-HBs status should be followed carefully by means of LFT with or without HBV DNA testing, and be treated with NUCs upon confirmation of HBVr. Liver fibrosis assessment was included in the algorithm as fibrosis or cirrhosis. Low-risk HBsAg + without advanced fibrosis or cirrhosis should be monitored with ALT testing every three months. Moderate and low risks $\mathrm{HBsAg}$ - anti-HBc + patients without advanced fibrosis or cirrhosis should be monitored with ALT testing every three months

patients with advanced fibrosis and cirrhosis run a higher risk of mortality and morbidity with $\mathrm{HBVr}$. Also, new data on $\mathrm{HBVr}$ with $\mathrm{HBV} / \mathrm{HCV}$ coinfected patients treated with DAAs are included. The evolving data over the last few years have better elucidated the risk of $\mathrm{HBVr}$ in $\mathrm{HBsAg}$-negative, anti-HBc positive patients. Hence, pre-emptive NUCs are now recommended for HBsAg-negative, anti-HBc positive patients in the moderate-risk and high-risk groups.

\section{Recommendations}

\section{Definition}

\subsection{Reactivation of HBV}

1.1.1. Exacerbation of chronic HBV infection ( $\mathrm{HBsAg}+)$

- $\geq 2 \log$ increase in HBV DNA levels from baseline levels

[Grading: evidence-II-2, recommendation -1] 
- Detection of HBV DNA with level $>100 \mathrm{IU} / \mathrm{ml}$ in a person with undetectable HBV DNA at baseline

\section{[Grading: evidence-II-2, recommendation -1]}

1.1.2. Reactivation of past $\mathrm{HBV}$ infection (HBsAg negative, anti-HBc positive) after the start of immunosuppressive therapy

- Reverse HBsAg seroconversion, HBsAg-negative becomes HBsAg-positive

[Grading: evidence-II-2, recommendation -1]

- Appearance of HBV DNA in absence of HBsAg, HBV DNA-undetectable becomes HBV DNA-detectable

[Grading: evidence-II-2, recommendation -1]

2. Who should be screened?

All patients planned to receive immunosuppressive therapy should be screened.

[Grading: evidence-II-2, recommendation -1]

3. What will be screened?

3.1 Screening should include HBsAg, anti-HBs and anti-HBc.

[Grading: evidence-II-2, recommendation -1]

3.2 For those HBsAg-positive patients, additional test for quantitative HBV DNA and HBsAg should be considered.

\section{[Grading: evidence-II-2, recommendation -1]}

3.3 All HBsAg positive and HBsAg negative but anti$\mathrm{HBc}$ positive patients should have the degree of liver fibrosis assessed by a hepatologist. [Grading: evidence-III, recommendation -2]

4. Management

4.1 It is mandatory and life-saving to administer preemptive NUCs promptly to the followings:

- In high-risk group, all $\mathrm{HBsAg}$ positive or $\mathrm{HBsAg}$ negative but anti-HBc positive patients

[Grading: evidence -I, recommendation -1]

- In moderate-risk group, all HBsAg positive and those who are anti-HBc positive with advanced liver fibrosis or cirrhosis

\section{[Grading: evidence-II-2, recommendation -1]}

4.2 It is essential to administer pre-emptive NUCs to the following:

- In low-risk group, all HBsAg positive or HBsAg negative but anti-HBc positive with advanced liver fibrosis or cirrhosis [Grading: evidence - II-2, recommendation -1]

4.3 Preferred NUCs include entecavir, tenofovir and TAF

[Grading: evidence - I, recommendation -1]
4.4 Termination of NUCs should be considered 6 months after the completion of immunosuppressive therapy:

- For HBsAg-positive patients, without advanced liver fibrosis or cirrhosis

[Grading: evidence-II-2, recommendation -1]

- For HBsAg-positive patients with low level of HBV DNA $(<2000 \mathrm{IU} / \mathrm{ml})$ before initiation of NUCs

[Grading: evidence-II-2, recommendation -1]

- For those who remain HBsAg negative but anti-HBc positive

[Grading: evidence-II-2, recommendation -1]

5.1 Liver function test* should be monitored every 12-weekly

5.1 In moderate-risk group, HBsAg negative but anti$\mathrm{HBc}$-positive patients with no advanced liver fibrosis or cirrhosis

[Grading: evidence-II-2, recommendation -1]

5.2 In low-risk group, HBsAg positive or HBsAg negative but anti-HBc positive with no advanced liver fibrosis or cirrhosis

[Grading: evidence-II-2, recommendation -2]

5.3 If ALT $>2 \times$ baseline, check HBsAg, HBV DNA and treat with NUCs for HBsAg seroreversion and/or HBV DNA detectable

[Grading: evidence $-I I-2$, recommendation -1]

*Liver function test includes ALT, AST, bilirubin, albumin, globulin

Conflict of Interest Ming-Lung Yu received research grants from Abbott, BMS, Gilead and Merck and received fees for being a speaker/consultant from Abbvie, Abbott, BMS, Gilead, Merck, Ipsen and Roche. Grace Wong has served as an advisory committee member for Gilead Sciences and Janssen, as a speaker for Abbott, Abbvie, Bristol-Myers Squibb, Echosens, Furui, Gilead Sciences, Janssen and Roche, and received research grant from Gilead Sciences. Alexander Thompson has served as an advisory committee member for Gilead Sciences, Abbvie, Roche, BMS, Merck, Immunocore, Janssen, Assembly Biosciences, Arbutus, Eisai, Ipsen and Bayer, as a speaker for Gilead Sciences, Abbvie, Roche, BMS, and received research grant from Gilead Sciences, Merck, BMS, Abbvie. Jin-Lin Hou received grants and personal fees from Bristol-Myers Squibb during the conduct of the study, and grants and personal fees from Bristol-Myers Squibb, GlaxoSmithKline, and Novartis. Teerha Piratvisuth received fees for being a speaker and advisory board member from Bayer, BMS, Gilead Sciences, and Eisai and received research support from Fibrogen, Gilead Sciences, Janssen and Roche. Ji-Dong Jia received consultation and speaker fees from Bristol-Myers Squibb, Gilead, Merck Sharp and Dohme, Novartis, and Roche. Ann-Lii Cheng received consultant fees from Novartis, Merck Serono, Eisai, Merck Sharp and Dohme, ONXEO, Bayer, BristolMyers Squibb, and Ono Pharmaceutical. Tony Mok received fees for 
being a speaker, consultant, and advisory board member from AstraZeneca, Roche/Genentech, Lilly, Bristol Myers Squibb, Boehringer Ingelheim, Novartis, Merck Sharp \& Dohme, Pfizer, Merck Serono, SFJ Pharmaceuticals Group, ACEA Biosciences, Vertex, Celgene, Ignyta, Fishawack Facilitate Ltd, Takeda, Janssen, Hutchison MediPharma, and received grants from AstraZeneca, Boehringer Ingelheim, Pfizer, Novartis, SFJ Pharmaceuticals Group, Roche, Merck Sharp \& Dohme, Clovis Oncology, Bristol Myers Squibb, Xcovery. Diana A. Payawal has served as an advisory committee member for Mylan Pharmaceutical, as a speaker for Gilead Sciences, Mylan Pharmaceuticals, Echosense, Getz and Abbott. Tawesak Tanwandee received grants from Bristol-Myers Squibb and Merck. Masao Omata received fees for being a speaker, consultant, and advisory board member from Bayer, Boehringer Ingelheim, Bristol-Myers Squibb, Otsuka, Astellas, Gilead Sciences, Chugai, Mitsubishi Tanabe, Kyorin, Merck Sharp and Dohme, Dainippon Sumitomo, Vertex Pharmaceuticals, Takeda, Merck Serono, and Zeria. George Lau, Hasmik Ghazinian, Masashi Mizokami, Gregory Cheng, Guo-Feng Chen, Zhen-Wen Liu, Oidov Baatarkhuu, Woon Leung Ng, Patrick Lau, Jer-Ming Chang, Saeed Hamid, A. Kadir Dokmeci, Rino A Gani, Diana A. Payawal, Pierce Chow, Joong-Won Park, Simone I Strasser, Rosmawaiti Mohamed, Khin Maung Win, Shiv Kumar Sari declare that they have no conflict of interest.

\section{References}

1. Wands JR, Chura CM, Roll FJ, Maddrey WC. Serial studies of hepatitis-associated antigen and antibody in patients receiving antitumor chemotherapy for myeloproliferative and lymphoproliferative disorders. Gastroenterology. 1975;68(1):105-12.

2. Hoofnagle JH, Dusheiko GM, Schafer DF, Jones EA, Micetich KC, Young RC, Costa J. Reactivation of chronic hepatitis $\mathrm{B}$ virus infection by cancer chemotherapy. Ann Intern Med. 1982;96(4):447-9.

3. Lok AS, Liang RH, Chiu EK, Wong KL, Chan TK, Todd D. Reactivation of hepatitis $\mathrm{B}$ virus replication in patients receiving cytotoxic therapy. Report of a prospective study. Gastroenterology. 1991;100(1):182-8.

4. Lau G, Liang R, Chiu EK, Lee CK, Lam SK. Hepatic events after bone marrow transplantation in patients with hepatitis $\mathrm{B}$ infection: a case controlled study. Bone Marrow Transplant. 1997;19(8):795-9.

5. Lau G. Hepatitis B reactivation after chemotherapy: two decades of clinical research. Hepatol Int. 2008;2(2):152-62.

6. Liang R, Lau GK, Kwong YL. Chemotherapy and bone marrow transplantation for cancer patients who are also chronic hepatitis B carriers: a review of the problem J. Clin Oncol. 1999; 17(1):394-8.

7. Hui CK, Cheung WW, Zhang HY, Au WY, Yueng YH, Leung AY, Leung N, Luk JM, Lie AK, Kwong YL, Liang R, Lau GK. Kinetics and risk of de novo hepatitis B infection in HBsAgnegative patients undergoing cytotoxic chemotherapy. Gastroenterology. 2006;131(1):59-68.

8. Yeo W, Chan TC, Leung NW, Lam WY, Mo FK, Chu MT, Chan HL, Hui EP, Lei KI, Mok TS, Chan PK. Hepatitis B virus reactivation in lymphoma patients with prior resolved hepatitis B undergoing anticancer therapy with or without rituximab. J Clin Oncol. 2009;27(4):605-11.

9. Matsue K, Kimura S, Takanashi Y, Iwama K, Fujiwara H, Yamakura M, Takeuchi M. Reactivation of hepatitis B virus after rituximab-containing treatment in patients with $\mathrm{CD} 20$-positive B-cell lymphoma. Cancer. 2010;116(20):4769-76.

10. Huang YH, Hsiao LT, Hong YC, Chiou TJ, Yu YB, Gau JP, Liu CY, Yang MH, Tzeng CH, Lee PC, Lin HC, Lee SD. Randomized controlled trial of entecavir prophylaxis for rituximab-associated hepatitis B virus reactivation in patients with lymphoma and resolved hepatitis B. J Clin Oncol. 2013;31(22):2765-72.

11. Seto WK, Chan TS, Hwang YY, Wong DK, Fung J, Liu KS, Gill H, Lam YF, Lie AK, Lai CL, Kwong YL, Yuen MF. Hepatitis B reactivation in patients with previous hepatitis $\mathrm{B}$ virus exposure undergoing rituximab-containing chemotherapy for lymphoma: a prospective study. J Clin Oncol. 2014;32(33):3736-43.

12. Hsu C, Tsou HH, Lin SJ, Wang MC, Yao M, Hwang WL, Kao WY, Chiu CF, Lin SF, Lin J, Chang CS, Tien HF, Liu TW, Chen PJ, Cheng AL, Taiwan Cooperative Oncology Group. Chemotherapy-induced hepatitis B reactivation in lymphoma patients with resolved HBV infection: a prospective study. Hepatology. 2014;59(6):2092-100.

13. Buti M, Manzano ML, Morillas RM, García-Retortillo M, Martín L, Prieto M, Gutiérrez ML, Suárez E, Gómez Rubio M, López J, Castillo P, Rodríguez M, Zozaya JM, Simón MA, Morano LE, Calleja JL, Yébenes M, Esteban R. Randomized prospective study evaluating tenofovir disoproxil fumarate prophylaxis against hepatitis B virus reactivation in anti-HBc-positive patients with rituximab-based regimens to treat hematologic malignancies: the Preblin study. PLoS ONE. 2017;12(9):e0184550.

14. Lau G, Yiu HH, Fong DY, Cheng HC, Au WY, Lai LS, Cheung M, Zhang HY, Lie A, Ngan R, Liang R. Early is superior to deferred preemptive lamivudine therapy for hepatitis B patients undergoing chemotherapy. Gastroenterology. 2003;125(6):1742-9.

15. Jang JW, Choi JY, Bae SH, Yoon SK, Chang UI, Kim CW, Cho SH, Han JY, Lee YS. A randomized controlled study of preemptive lamivudine in patients receiving transarterial chemo-lipiodolization. Hepatology. 2006;43(2):233-40.

16. Hsu C, Hsiung CA, Su IJ, Hwang WS, Wang MC, Lin SF, Lin $\mathrm{TH}$, Hsiao HH, Young JH, Chang MC, Liao YM, Li CC, Wu HB, Tien HF, Chao TY, Liu TW, Cheng AL, Chen PJ. A revisit of prophylactic lamivudine for chemotherapy-associated hepatitis B reactivation in non-Hodgkin's lymphoma: a randomized trial. Hepatology. 2008;47(3):844-53.

17. Reddy KR, Beavers KL, Hammond SP, Lim JK, Falck-Ytter YT, American Gastroenterological Association Institute. American Gastroenterological Association Institute guideline on the prevention and treatment of hepatitis B virus reactivation during immunosuppressive drug therapy. Gastroenterology. 2015;148(1):215-9.

18. Sarin SK, Kumar M, Lau G, Abbas Z, Chan HL, Chen CJ, Chen DS, Chen HL, Chen PJ, Chien RN, Dokmeci AK, Gane E, Hou JL, Jafri W, Jia J, Kim JH, Lai CL, Lee HC, Lim SG, Liu CJ, Locarnini S, Al Mahtab M, Mohamed R, Omata M, Park J, Piratvisuth T, Sharma BC, Sollano J, Wang FS, Wei L, Yuen MF, Zheng SS, Kao JH. Asian-Pacific clinical practice guidelines on the management of hepatitis B: a 2015 update. Hepatol Int. 2016;10(1):1-98.

19. European Association for the Study of the Liver. EASL 2017 Clinical Practice Guidelines on the management of hepatitis B virus infection. J Hepatol. 2017;67(2):370-98.

20. Terrault NA, Lok ASF, McMahon BJ, Chang KM, Hwang JP, Jonas MM, Brown RS Jr, Bzowej NH, Wong JB. Update on prevention, diagnosis, and treatment of chronic hepatitis B: AASLD 2018 hepatitis B guidance. Hepatology. 2018;67(4):1560-99.

21. Loomba R, Liang TJ. Hepatitis B reactivation associated with immune suppressive and biological modifier therapies: current concepts, management strategies, and future directions. Gastroenterology. 2017;152(6):1297-309.

22. Sarin SK, Choudhury A, Sharma MK, Maiwall R, Al Mahtab M, Rahman S, APASL ACLF Research Consortium (AARC) for APASL ACLF working Party, et al. Acute-on-chronic 
liver failure: consensus recommendations of the Asian Pacific association for the study of the liver (APASL): an update. Hepatol Int. 2019;13(4):353-90. https://doi.org/10.1007/ s12072-019-09946-3.

23. Uhm J, Kim S-H, Oh S, et al. High incidence of hepatitis B viral reactivation in chronic myeloid leukemia patients treated with tyrosine kinase inhibitors. Blood. 2018;132(Supplement 1):3010.

24. Wang YH, Liang JD, Sheng WH, Tien FM, Chen CY, Tien HF. Hepatitis $\mathrm{B}$ reactivation during treatment of tyrosine kinase inhibitors-Experience in 142 adult patients with chronic myeloid leukemia. Leuk Res. 2019;81:95-7.

25. Yao ZH, Liao WY, Ho CC, Chen KY, Shih JY, Chen JS, Lin ZZ, Lin CC, Yang JC, Yu CJ. Incidence of hepatitis B reactivation during epidermal growth factor receptor tyrosine kinase inhibitor treatment in non-small-cell lung cancer patients. Eur J Cancer. 2019;117:107-15.

26. Atteya A, Ahmad A, Daghstani D, Mushtaq K, Yassin MA. Evaluation of hepatitis $\mathrm{B}$ reactivation among patients with chronic myeloid leukemia treated with tyrosine kinase inhibitors. Cancer Control. 2020. https://doi.org/10.1177/1073274820976594.

27. Pu D, Yin L, Zhou Y, Li W, Huang L, Cai L, Zhou Q. Safety and efficacy of immune checkpoint inhibitors in patients with HBV/ $\mathrm{HCV}$ infection and advanced-stage cancer: a systematic review. Medicine (Baltimore). 2020;99(5):19013.

28. Zhang X, Zhou Y, Chen C, Fang W, Cai X, Zhang X, Zhao M, Zhang B, Jiang W, Lin Z, Ma Y, Yang Y, Huang Y, Zhao H, Xu $\mathrm{R}$, Hong S, Zhang L. Hepatitis B virus reactivation in cancer patients with positive Hepatitis B surface antigen undergoing PD-1 inhibition. J Immunother Cancer. 2019;7(1):322.

29. Lee PC, Chao Y, Chen MH, Lan KH, Lee IC, Hou MC, Huang YH. Risk of HBV reactivation in patients with immune checkpoint inhibitor-treated unresectable hepatocellular carcinoma. $\mathbf{J}$ Immunother Cancer. 2020;8(2):e001072.

30. Lan JL, Chen YM, Hsieh TY, Chen YH, Hsieh CW, Chen DY, Yang SS. Kinetics of viral loads and risk of hepatitis B virus reactivation in hepatitis $\mathrm{B}$ core antibody-positive rheumatoid arthritis patients undergoing anti-tumour necrosis factor alpha therapy. Ann Rheum Dis. 2011;70(10):1719-25.

31. Tamori A, Koike T, Goto H, Wakitani S, Tada M, Morikawa H, Enomoto M, Inaba M, Nakatani T, Hino M, Kawada N. Prospective study of reactivation of hepatitis B virus in patients with rheumatoid arthritis who received immunosuppressive therapy: evaluation of both HBsAg-positive and HBsAg-negative cohorts. J Gastroenterol. 2011;46(4):556-64.

32. Ryu HH, Lee EY, Shin K, Choi IA, Lee YJ, Yoo B, Park MC, Park YB, Bae SC, Yoo WH, Kim SI, Lee EB, Song YW. Hepatitis $\mathrm{B}$ virus reactivation in rheumatoid arthritis and ankylosing spondylitis patients treated with anti-TNF $\alpha$ agents: a retrospective analysis of 49 cases. Clin Rheumatol. 2012;31(6):931-6.

33. Tan J, Zhou J, Zhao P, Wei J. Prospective study of HBV reactivation risk in rheumatoid arthritis patients who received conventional disease-modifying antirheumatic drugs. Clin Rheumatol. 2012;31(8):1169-75.

34. Lee YH, Bae SC, Song GG. Hepatitis B virus reactivation in HBsAg-positive patients with rheumatic diseases undergoing anti-tumor necrosis factor therapy or DMARDs. Int J Rheum Dis. 2013;16(5):527-31.

35. Chen MH, Chen MH, Liu CY, Tsai CY, Huang DF, Lin HY, Lee $\mathrm{MH}$, Huang YH. Hepatitis B virus reactivation in rheumatoid arthritis patients undergoing biologics treatment. J Infect Dis. 2017;215(4):566-73.

36. Bersoff-Matcha SJ, Cao K, Jason M, Ajao A, Jones SC, Meyer T, Brinker A. Hepatitis B virus reactivation associated with directacting antiviral therapy for chronic hepatitis $\mathrm{C}$ virus: a review of cases reported to the U.S. food and drug administration adverse event reporting system. Ann Intern Med. 2017;166(11):792-8.
37. Gane EJ, Hyland RH, An D, Svarovskaia ES, Brainard D, McHutchison JG. Ledipasvir and sofosbuvir for HCV infection in patients coinfected with HBV. Antivir Ther. 2016;21:605-9.

38. Doi A, Sakamori R, Tahata Y, Urabe A, Morishita N, Yamada R, et al. Frequency of, and factors associated with, hepatitis B virus reactivation in hepatitis $\mathrm{C}$ patients treated with all-oral direct-acting antivirals: analysis of a Japanese prospective cohort. Hepatol Res. 2017;47:1438-44.

39. Kawagishi N, Suda G, Onozawa M, Kimura M, Maehara O, Ohara $\mathrm{M}$, et al. Comparing the risk of hepatitis B virus reactivation between direct-acting antiviral therapies and interferonbased therapies for hepatitis C. J Viral Hepat. 2017;24:1098-106.

40. Wang C, Ji D, Chen J, Shao Q, Li B, Liu J, et al. Hepatitis due to reactivation of hepatitis $\mathrm{B}$ virus in endemic areas among patients with hepatitis $\mathrm{C}$ treated with direct-acting antiviral agents. Clin Gastroenterol Hepatol. 2017;15:132-6.

41. Tamori A, Abiru S, Enomoto H, Kioka K, Korenaga M, Tani J, et al. Low incidence of hepatitis $\mathrm{B}$ virus reactivation and subsequent hepatitis in patients with chronic hepatitis $\mathrm{C}$ receiving direct-acting antiviral therapy. J Viral Hepat. 2018;25:608-11.

42. Liu CJ, Chuang WL, Sheen IS, Wang HY, Chen CY, Tseng KC, et al. Efficacy of Ledipasvir and Sofosbuvir treatment of HCV infection in patients coinfected with HBV. Gastroenterology. 2018;154:989-97.

43. Yeh ML, Huang CF, Hsieh MH, Ko YM, Chen KY, Liu TW, et al. Reactivation of hepatitis $\mathrm{B}$ in patients of chronic hepatitis $\mathrm{C}$ with hepatitis $\mathrm{B}$ virus infection treated with direct acting antivirals. J Gastroenterol Hepatol. 2017;32:1754-62.

44. Liu CH, Liu CJ, Su TH, et al. Hepatitis B virus reactivation in patients receiving interferon-free direct-acting antiviral agents for chronic hepatitis $\mathrm{C}$ virus infection. Open Forum Infect Dis. 2017. https://doi.org/10.1093/ofid/ofx028.

45. Lee SW, Lee TY, Yang SS, Peng YC, Yeh HZ, Chang CS. Prevalence of hepatitis B reactivation among Chinese individuals with chronic hepatitis $\mathrm{C}$ treated with pan-oral direct-acting antivirals. Gastroenterol Res. 2018;11(2):124-9.

46. Yeh ML, Huang CF, Huang CI, et al. Hepatitis B-related outcomes following direct-acting antiviral therapy in Taiwanese patients with chronic HBV/HCV co-infection. J Hepatol. 2020;73(1):62-71.

47. Sulkowski MS, Chuang WL, Kao JH, et al. No evidence of reactivation of hepatitis B virus among patients treated with Ledipasvir-Sofosbuvir for hepatitis $C$ virus infection. Clin Infect Dis. 2016;63(9):1202-4.

48. Ogawa E, Furusyo N, Murata M, Toyoda K, Hayashi T, Ura K. Potential risk of HBV reactivation in patients with resolved HBV infection undergoing direct-acting antiviral treatment for HCV. Liver Int. 2018;38:76-83.

49. Toy M, Hutton DW, So SK. Cost-effectiveness and cost thresholds of generic and brand drugs in a national chronic hepatitis B treatment program in China. PLoS ONE. 2015;10(11):e0139876.

50. Xiao Y, Thompson AJ, Howell J. Point-of-care tests for hepatitis b: an overview. Cells. 2020;9(10):2233. https://doi.org/10.3390/ cells9102233.PMID:33023265;PMCID:PMC7650625.

51. Guyatt GH, Oxman AD, Vist GE, Kunz R, Falck-Ytter Y, AlonsoCoello P, Schünemann HJ, GRADE Working Group. GRADE: an emerging consensus on rating quality of evidence and strength of recommendations. BMJ. 2008;336(7650):924-6.

52. Tsukuda S, Watashi K. Hepatitis B virus biology and life cycle. Antiviral Res. 2020;182: 104925. https://doi.org/10.1016/j.antiv iral.2020.104925.

53. Hui CK, Bowden S, Luk JM, Fong DYT, Zhang HY, Jackson K, Au WY, Lie A, Chim CS, Kwong YL, Liang R, Locarnini S, Lau G. Intrahepatic hepatitis B virus covalently closed circular DNA predicts post-chemotherapy Hepatitis B reactivation. Blood. $2005 ; 15: 2616-7$ 
54. Lang J, Neumann-Haefelin C, Thimme R. Immunological cure of HBV infection. Hepatol Int. 2019;13(2):113-24.

55. Lau G, Suri D, Liang R, Chokshi S, Thomas MG, Nanji A, Yuen ST, Williams R, Naoumov NV. Resolution of chronic hepatitis $\mathrm{B}$ and Anti-HBs seroconversion in man by adoptive transfer of immunity to hepatitis B core antigen. Gastroenterology. 2002;122:614-24.

56. Evens AM, Jovanovic BD, Su YC, Raisch DW, Ganger D, Belknap SM, Dai MS, Chiu BC, Fintel B, Cheng Y, Chuang SS, Lee MY, Chen TY, Lin SF, Kuo CY. Rituximab-associated hepatitis B virus (HBV) reactivation in lymphoproliferative diseases: meta-analysis and examination of FDA safety reports. Ann Oncol. 2011;22(5):1170-80.

57. Dong HJ, Ni LN, Sheng GF, et al. Risk of hepatitis B virus (HBV) reactivation in non-Hodgkin lymphoma patients receiving rituximab-chemotherapy: a meta-analysis. J Clin Virol. 2013;57(3):209-14.

58. Jang JW, Choi JY, Bae SH, Kim CW, Yoon SK, Cho SH, Yang JM, Ahn BM, Lee CD, Lee YS, Chung KW, Sun HS. Transarterial chemo-lipiodolization can reactivate hepatitis B virus replication in patients with hepatocellular carcinoma. J Hepatol. 2004;41(3):427-35.

59. Huang L, Jiang S, Shi Y. Tyrosine kinase inhibitors for solid tumors in the past 20 years (2001-2020). J Hematol Oncol. 2020;13(1):143.

60. Li J, Huang B, Li Y, Zheng D, Zhou Z, Liu J. Hepatitis B virus reactivation in patients with multiple myeloma receiving bortezomib-containing regimens followed by autologous stem cell transplant. Leuk Lymphoma. 2015;56(6):1710-7.

61. Tur-Kaspa R, Shaul Y, Moore DD, Burk RD, Okret S, Poellinger L, Shafritz DA. The glucocorticoid receptor recognizes a specific nucleotide sequence in hepatitis B virus DNA causing increased activity of the HBV enhancer. Virology. 1988;167(2):630-3.

62. Cheng X, Uchida T, Xia Y, Umarova R, Liu CJ, Chen PJ, Gaggar A, Suri V, Mücke MM, Vermehren J, Zeuzem S, Teraoka Y, Osawa M, Aikata H, Tsuji K, Mori N, Hige S, Karino Y, Imamura M, Chayama K, Liang TJ. Diminished hepatic IFN response following $\mathrm{HCV}$ clearance triggers $\mathrm{HBV}$ reactivation in coinfection. J Clin Invest. 2020;130(6):3205-20.

63. Koh C, Canini L, Dahari H, Zhao X, Uprichard SL, Haynes-Williams V, Winters MA, Subramanya G, Cooper SL, Pinto P, Wolff EF, Bishop R, Ai Thanda Han M, Cotler SJ, Kleiner DE, Keskin O, Idilman R, Yurdaydin C, Glenn JS, Heller T. Oral prenylation inhibition with lonafarnib in chronic hepatitis D infection: a proof-of-concept randomised, double-blind, placebo-controlled phase 2A trial. Lancet Infect Dis. 2015;15(10):1167-74.

64. Dore GJ, Soriano V, Rockstroh J, Kupfer B, Tedaldi E, Peters L, Neuhaus J, Puoti M, Klein MB, Mocroft A, Clotet B, Lundgren JD, SMART INSIGHT study group. Frequent hepatitis B virus rebound among HIV-hepatitis B virus-coinfected patients following antiretroviral therapy interruption. AIDS. 2010;24(6):857-65.

65. Nakamoto S, Kanda T, Nakaseko C, Sakaida E, Ohwada C, Takeuchi M, Takeda Y, Mimura N, Iseki T, Wu S, Arai M, Imazeki F, Saito K, Shirasawa H, Yokosuka O. Reactivation of hepatitis B virus in hematopoietic stem cell transplant recipients in Japan: efficacy of nucleos(t)ide analogues for prevention and treatment. Int J Mol Sci. 2014;15(11):21455-67.

66. Lau GK, He ML, Fong DY, Bartholomeusz A, Au WY, Lie AK, Locarnini S, Liang R. Preemptive use of lamivudine reduces hepatitis B exacerbation after allogeneic hematopoietic cell transplantation. Hepatology. 2002;36(3):702-9.

67. Yeo W, Chan PK, Ho WM, et al. Lamivudine for the prevention of hepatitis B virus reactivation in hepatitis B s-antigen seropositive cancer patients undergoing cytotoxic chemotherapy. J Clin Oncol. 2004;22(5):927-34.
68. Kim MK, Ahn JH, Kim SB, et al. Hepatitis B reactivation during adjuvant anthracycline-based chemotherapy in patients with breast cancer: a single institution's experience. Korean J Intern Med. 2007;22(4):237-43.

69. Paul S, Saxena A, Terrin N, Viveiros K, Balk EM, Wong JB. Hepatitis B virus reactivation and prophylaxis during solid tumor chemotherapy: a systematic review and meta-analysis. Ann Intern Med. 2016;164(1):30-40.

70. Cheng AL, Hsiung CA, Su IJ, et al. Steroid-free chemotherapy decreases risk of hepatitis B virus (HBV) reactivation in HBVcarriers with lymphoma. Hepatology. 2003;37(6):1320-8.

71. Chen CY, Tien FM, Cheng A, Huang SY, Chou WC, Yao M, Tang JL, Tien HF, Sheng WH. Hepatitis B reactivation among 1962 patients with hematological malignancy in Taiwan. BMC Gastroenterol. 2018;18(1):6.

72. Yeo W, Lam KC, Zee B, et al. Hepatitis B reactivation in patients with hepatocellular carcinoma undergoing systemic chemotherapy. Ann Oncol. 2004;15(11):1661-6.

73. Lee HJ, Kim DY, Keam B, et al. Lamivudine prophylaxis for hepatitis B virus carrier patients with breast cancer during adjuvant chemotherapy. Breast Cancer. 2014;21(4):387-93.

74. Jang JW, Choi JY, Bae SH, et al. A randomized controlled study of preemptive lamivudine in patients receiving transarterial chemolipiodolization. Hepatology. 2006;43(2):233-40.

75. Jang JW, Kwon JH, You CR, et al. Risk of HBV reactivation according to viral status and treatment intensity in patients with hepatocellular carcinoma. Antivir Ther. 2011;16(7):969-77.

76. Kuo MH, Tseng CW, Lu MC, Tung CH, Tseng KC, Huang KY, Lee $\mathrm{CH}$, Lai NS. Risk of hepatitis B virus reactivation in rheumatoid arthritis patients undergoing tocilizumab-containing treatment. Dig Dis Sci. 2021;2:1-9.

77. Sonneveld MJ, Murad SD, van der Eijk AA, de Man RA. Fulminant liver failure due to hepatitis $B$ reactivation during treatment with tocilizumab. ACG Case Rep J. 2019;6(12):e00243.

78. APASL Covid-19 Task Force, Lau G, Sharma M. Clinical practice guidance for hepatology and liver transplant providers during the COVID-19 pandemic: APASL expert panel consensus recommendations. Hepatol Int. 2020;14(4):415-28.

79. Seto WK, Chan TS, Hwang YY, Wong DK, Fung J, Liu KS, Gill H, Lam YF, Lau EHY, Cheung KS, Lie AKW, Lai CL, Kwong YL, Yuen MF. Hepatitis B reactivation in occult viral carriers undergoing hematopoietic stem cell transplantation: a prospective study. Hepatology. 2017;65(5):1451-61.

80. Wu T, Wu N, Ma YX, Wu J, Gao Y, Pan XB. Role of hepatitis $\mathrm{B}$ antibody in predicting reactivation of resolved hepatitis B virus infection in leukemia patients. Antiviral Res. 2020;177:104765.

81. Nishikawa K, Kimura K, Kanda Y, Sugiyama M, Kakihana K, Doki N, Ohashi K, Bae SK, Takahashi K, Ishihara Y, Mizuno I, Onishi Y, Onozawa M, Onizuka M, Yamamoto M, Ishikawa T, Inoue K, Kusumoto S, Hashino S, Saito H, Kanto T, Sakamaki H, Mizokami M. A prospective trial of vaccine to prevent hepatitis $B$ virus reactivation after hematopoietic stem cell transplantation. Bone Marrow Transplant. 2020;55(7):1388-98.

82. Paul S, Dickstein A, Saxena A, Terrin N, Viveiros K, Balk EM, Wong JB. Role of surface antibody in hepatitis B reactivation in patients with resolved infection and hematologic malignancy: a meta-analysis. Hepatology. 2017;66(2):379-88.

83. Kusumoto S, Arcaini L, Hong X, Jin J, Kim WS, Kwong YL, Peters MG, Tanaka Y, Zelenetz AD, Kuriki H, Fingerle-Rowson G, Nielsen T, Ueda E, Piper-Lepoutre H, Sellam G, Tobinai K. Risk of HBV reactivation in patients with B-cell lymphomas receiving obinutuzumab or rituximab immunochemotherapy. Blood. 2019;133(2):137-46.

84. Wong GL, Wong VW, Yuen BW, Tse YK, Yip TC, Luk HW, Lui GC, Chan HL. Risk of hepatitis B surface antigen seroreversion 
after corticosteroid treatment in patients with previous hepatitis B virus exposure. J Hepatol. 2020;72(1):57-66.

85. Lau GK, Leung YH, Fong DY, Au WY, Kwong YL, Lie A, Hou JL, Wen YM, Nanj A, Liang R. High hepatitis B virus (HBV) DNA viral load as the most important risk factor for HBV reactivation in patients positive for HBV surface antigen undergoing autologous hematopoietic cell transplantation. Blood. 2002;99(7):2324-30.

86. Kanda T, Lau GKK, Wei L, Moriyama M, Yu ML, Chuang WL, Ibrahim A, Lesmana CRA, Sollano J, Kumar M, Jindal A, Sharma BC, Hamid SS, Kadir Dokmeci A, Mamun-Al-Mahtab, McCaughan GW, Wasim J, Crawford DHG, Kao JH, Ooka Y, Yokosuka O, Sarin SK, Omata M. APASL HCV guidelines of virus-eradicated patients by DAA on how to monitor HCC occurrence and HBV reactivation. Hepatol Int. 2019;13(6):649-61.

87. Yu ML, Chen PJ, Dai CY, Hu TH, Huang CF, Huang YH, Hung $\mathrm{CH}$, Lin CY, Liu CH, Liu CJ, Peng CY, Lin HC, Kao JH, Chuang WL. 2020 Taiwan consensus statement on the management of hepatitis C: part (II) special populations. J Formos Med Assoc. 2020;119(7):1135-57.

88. Chen G, Wang C, Chen J, Ji D, Wang Y, Wu V, Karlberg J, Lau G. Hepatitis B reactivation in hepatitis B and C coinfected patients treated with antiviral agents: a systematic review and meta-analysis. Hepatology. 2017;66(1):13-26.

89. Perrillo RP, Gish R, Falck-Ytter YT. American Gastroenterological Association Institute technical review on prevention and treatment of hepatitis B virus reactivation during immunosuppressive drug therapy. Gastroenterology. 2015;148(1):221-44.

90. Mallet V, van Bömmel F, Doerig C, Pischke S, Hermine O, Locasciulli A, Cordonnier C, Berg T, Moradpour D, Wedemeyer H, Ljungman P, ECIL-5. Management of viral hepatitis in patients with haematological malignancy and in patients undergoing haemopoietic stem cell transplantation: recommendations of the 5th European Conference on Infections in Leukaemia (ECIL-5). Lancet Infect Dis. 2016;16(5):606-17.

91. Doyle J, Raggatt M, Slavin M, McLachlan SA, Strasser SI, Sasadeusz JJ, Howell J, Hajkowicz K, Nandurkar H, Johnston A, Bak N, Thompson AJ. Hepatitis B management during immunosuppression for haematological and solid organ malignancies: an Australian consensus statement. Med J Aust. 2019;210(10):462-8.

92. Pei SN, Ma MC, Wang MC, Kuo CY, Rau KM, Su CY, Chen $\mathrm{CH}$. Analysis of hepatitis B surface antibody titers in B cell lymphoma patients after rituximab therapy. Ann Hematol. 2012;91(7):1007-12.

93. Fang J, Li W, Tan Z, Li D. Comparison of prednisolone and lamivudine combined therapy with prednisolone monotherapy on carriers of hepatitis B virus with IgA nephropathy: a prospective cohort study. Int Urol Nephrol. 2014;46(1):49-56.

94. Loomba R, Rowley A, Wesley R, Liang TJ, Hoofnagle JH, Pucino F, Csako G. Systematic review: the effect of preventive lamivudine on hepatitis B reactivation during chemotherapy. Ann Intern Med. 2008;148(7):519-28.

95. Huang H, Li X, Zhu J, Ye S, Zhang H, Wang W, Wu X, Peng $\mathrm{J}$, Xu B, Lin Y, Cao Y, Li H, Lin S, Liu Q, Lin T. Entecavir vs lamivudine for prevention of hepatitis $\mathrm{B}$ virus reactivation among patients with untreated diffuse large B-cell lymphoma receiving R-CHOP chemotherapy: a randomized clinical trial. JAMA. 2014;312(23):2521-30.

96. Ho EY, Yau T, Rousseau F, Heathcote EJ, Lau GK. Preemptive adefovir versus lamivudine for prevention of hepatitis B reactivation in chronic hepatitis $\mathrm{B}$ patients undergoing chemotherapy. Hepatol Int. 2015;9(2):224-30.

97. Svicher V, Salpini R, Malagnino V, Piermatteo L, Alkhatib M, Cerva C, Sarmati L. New markers in monitoring the reactivation of hepatitis B virus infection in immunocompromised hosts. Viruses. 2019;11(9):783.

98. Wong GL. Non-invasive assessments for liver fibrosis: the crystal ball we long for. J Gastroenterol Hepatol. 2018;33:1009-15.

99. Loomba R, Adams LA. Advances in non-invasive assessment of hepatic fibrosis. Gut. 2020;69(7):1343-52.

100. Jia J, Hou J, Ding H, Chen G, Xie Q, Wang Y, Zeng M, Zhao J, Wang T, Hu X, Schuppan D. Transient elastography compared to serum markers to predict liver fibrosis in a cohort of Chinese patients with chronic hepatitis B. J Gastroenterol Hepatol. 2015;30(4):756-62.

101. Li Q, Chen L, Zhou Y. Diagnostic accuracy of liver stiffness measurement in chronic hepatitis B patients with normal or mildly elevated alanine transaminase levels. Sci Rep. 2018;8(1):5224.

102. Agbim U, Asrani SK. Non-invasive assessment of liver fibrosis and prognosis: an update on serum and elastography markers. Expert Rev Gastroenterol Hepatol. 2019;13(4):361-74.

103. Kaewdech A, Tangkijvanich P, Sripongpun P, Witeerungrot T, Jandee S, Tanaka Y, Piratvisuth T. Hepatitis B surface antigen, core-related antigen and HBV RNA: Predicting clinical relapse after NA therapy discontinuation. Liver Int. 2020;40(12):2961-71

104. Long M, Jia W, Li S, Jin L, Wu J, Rao N, Feng H, Chen K, Deng $\mathrm{H}$, Liu F, Su F, Song E. A single-center, prospective and randomized controlled study: can the prophylactic use of lamivudine prevent hepatitis B virus reactivation in hepatitis B s-antigen seropositive breast cancer patients during chemotherapy? Breast Cancer Res Treat. 2011;127(3):705-12.

105. Koo YX, Tay M, Teh YE, Teng D, Tan DS, Tan IB, Tai DW, Quek R, Tao M, Lim ST. Risk of hepatitis B virus (HBV) reactivation in hepatitis B surface antigen negative/hepatitis B core antibody positive patients receiving rituximab-containing combination chemotherapy without routine antiviral prophylaxis. Ann Hematol. 2011;90(10):1219-23.

106. Mori S. Past hepatitis B virus infection in rheumatoid arthritis patients receiving biological and/or nonbiological disease-modifying antirheumatic drugs. Mod Rheumatol. 2011;21(6):621-7.

107. Urata Y, Uesato R, Tanaka D, Kowatari K, Nitobe T, Nakamura $\mathrm{Y}$, Motomura S. Prevalence of reactivation of hepatitis B virus replication in rheumatoid arthritis patients. Mod Rheumatol. 2011;21(1):16-23.

108. Watanabe T, Fukae J, Fukaya S, Sawamukai N, Isobe M, Matsuhashi M, Shimizu M, Akikawa K, Tanimura K, Atsumi T, Koike $\mathrm{T}$. Incidence and risk factors for reactivation from resolved hepatitis B virus in rheumatoid arthritis patients treated with biological disease-modifying antirheumatic drugs. Int J Rheum Dis. 2019;22(4):574-82.

Publisher's Note Springer Nature remains neutral with regard to jurisdictional claims in published maps and institutional affiliations. 


\section{Authors and Affiliations}

\section{George Lau ${ }^{1,2}$ (I) Ming-Lung $\mathrm{Yu}^{3} \cdot$ Grace Wong $^{4} \cdot$ Alexander Thompson $^{5} \cdot$ Hasmik Ghazinian $^{6} \cdot$ Jin-Lin Hou ${ }^{7}$. Teerha Piratvisuth $^{8}$. Ji-Dong Jia ${ }^{9} \cdot$ Masashi Mizokami ${ }^{10}$. Gregory Cheng ${ }^{2,11}$. Guo-Feng Chen ${ }^{12}$. Zhen-Wen Liu ${ }^{13}$. Oidov Baatarkhuu ${ }^{14}$. Ann Lii Cheng ${ }^{15}$. Woon Leung $\mathrm{Ng}^{16}$. Patrick Lau ${ }^{1}$. Tony Mok ${ }^{17}$. Jer-Ming Chang ${ }^{18}$. Saeed Hamid ${ }^{19}$. A. Kadir Dokmeci ${ }^{20} \cdot$ Rino A. Gani $^{21}$ - Diana A. Payawal ${ }^{22}$ - Pierce Chow ${ }^{23}$. Joong-Won Park ${ }^{24}$. Simone I. Strasser ${ }^{25} \cdot$ Rosmawaiti Mohamed ${ }^{26} \cdot$ Khin Maung Win $^{27} \cdot$ Tanwandee Tawesak $^{28} \cdot$ Shiv Kumar Sarin $^{29}$. Masao Omata ${ }^{30,31}$}

1 Humanity and Health Clinical Trial Center, Humanity and Health Medical Group, Hong Kong SAR, China

2 The Fifth Medical Center of Chinese, PLA General Hospital, Beijing 100039, China

3 Hepatitis Center and Hepatobiliary Division, Department of Internal Medicine, Kaohsiung Medical University Hospital, Kaohsiung Medical University, No. 100, Tz-You 1st Rd, Chinese Taipei, Kaohsiung, Taiwan

4 Department of Medicine and Therapeutics, The Chinese University of Hong Kong, Hong Kong SAR, China

5 St Vincent's Hospital, Melbourne, VIC, Australia

6 Department of Hepatology, Nork Clinical Hospital of Infectious Diseases, Yerevan, Armenia

7 Department of Infectious Diseases, Institute of Hepatology, Nanfang Hospital, Southern Medical University, Guangzhou, China

8 Department of Medicine, NKC Institute of Gastroenterology and Hepatology, Songklanagarind Hospital, Prince of Songkla University, Hat Yai, Thailand

9 Liver Research Center, Beijing Friendship Hospital, Beijing, China

10 Genome Medical Science Project, National Center for Global Health and Medicine, Ichikawa, Japan

11 Faculty of Health Science, Macau University, Macau SAR, China

12 Department of Liver Diseases, Fifth Medical Center of Chinese, PLA General Hospital, Beijing, China

13 Research Center for Liver Transplantation, Fifth Medical Center of Chinese PLA General Hospital, Beijing, China

14 Department of Infectious Diseases, Mongolian National University of Medical Sciences, Ulaanbaatar, Mongolia

15 Department of Internal Medicine, National Taiwan University Hospital, Taipei, Taiwan

16 Department of Medicine, United Christian Hospital, Hong Kong SAR, China
17 Department of Clinical Oncology, State Key Laboratory of South China, Chinese University of Hong Kong, Hong Kong SAR, China

18 Division of Nephrology, Kaohsiung Medical University Hospital, Kaohsiung Medical University, Kaohsiung, Taiwan

19 Department of Medicine, Aga Khan University and Hospital, Stadium Road, Karachi 74800, Pakistan

20 Department of Gastroenterology, Ankara University School of Medicine, Ankara, Turkey

21 Liver Transplantation Team, Ciptomangunkusumo Hospital, Jakarta, Indonesia

22 Department of Medicine, Cardinal Santos Medical Center, Mandaluyong, Metro, Manila, Philippines

23 Department of Hepatopancreatobiliary and Transplant Surgery, Singapore General Hospital, Singapore, Singapore

24 Center for Liver Cancer, Research Institute and Hospital, National Cancer Center, Goyang, Korea

25 AW Morrow Gastroenterology and Liver Centre, Royal Prince Alfred Hospital, Sydney, Australia

26 Department of Medicine, University Malaya Medical Centre, Kuala Lumpur, Malaysia

27 Yangon Gastroenterology and Liver Centre, Yangon, Myanmar

28 Division of Gastroenterology, Department of Medicine, Faculty of Medicine, Siriraj Hospital, Mahidol University, Bangkok, Thailand

29 Department of Hepatology, Institute of Liver and Biliary Sciences, New Delhi, India

30 Yamanashi Prefectural Central Hospital, 1-1-1 Fujimi, Kofu-shi, Yamanashi 400-8506, Japan

31 The University of Tokyo, 7-3-1 Hongo, Bunkyo-ku, Tokyo 113-8655, Japan 